\title{
The complex case of MACS J0717.5+3745 and its extended filament: intra-cluster light, galaxy luminosity function, and galaxy orientations
}

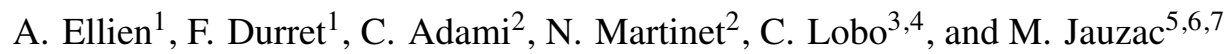 \\ 1 Sorbonne Université, CNRS, UMR 7095, Institut d'Astrophysique de Paris, 98bis Bd Arago, 75014 Paris, France \\ e-mail: ellien@iap.fr \\ 2 Aix-Marseille Univ., CNRS, CNES, LAM, Marseille, France \\ 3 Instituto de Astrofísica e Ciências do Espaço, Universidade do Porto, CAUP, Rua das Estrelas, 4150-762 Porto, Portugal \\ 4 Departamento de Física e Astronomia, Faculdade de Ciências, Universidade do Porto, Rua do Campo Alegre 687, \\ 4169-007 Porto, Portugal \\ 5 Centre for Extragalactic Astronomy, Department of Physics, Durham University, Durham DH1 3LE, UK \\ ${ }^{6}$ Institute for Computational Cosmology, Durham University, South Road, Durham DH1 3LE, UK \\ 7 Astrophysics and Cosmology Research Unit, School of Mathematical Sciences, University of KwaZulu-Natal, Durban 4041, \\ South Africa
}

Received 12 April 2019 / Accepted 22 May 2019

\begin{abstract}
Context. The properties of galaxies are known to be affected by their environment, but although galaxies in clusters and groups have been quite thoroughly investigated, little is known about galaxies belonging to filaments of the cosmic web, or about the properties of the filaments themselves.

Aims. Here we investigate the properties of the rich cluster MACS J0717.5+3745 and its extended filament by analyzing the distribution and fractions of intra-cluster light (ICL) in its core and by trying to detect intra-filament light (IFL) in the filament. We analyze the galaxy luminosity function (GLF) of the cluster core and of the filament. We also study the orientations of galaxies in the filament to better constrain the filament properties.

Methods. This work is based on Hubble Space Telescope (HST) archive data, both from the Hubble Frontier Fields in the F435W, F606W, F814W, and F105W bands, and from a mosaic of images in the F606W and F814W bands. The spatial distribution of the ICL was determined with our new wavelet-based software, DAWIS. The GLFs were extracted in the F606W and F814W bands, with a statistical subtraction of the background, and fit with Schechter functions. The galaxy orientations in the filaments were estimated with SExtractor after correction for the point spread function.

Results. We detect a large amount of ICL in the cluster core, but no IFL in the cosmic filament. The fraction of ICL in the core peaks in the F606W filter before decreasing with wavelength. Though relatively noisy, the GLFs in the filament are notably different from those of field galaxies, with a flatter faint end slope and an excess of bright galaxies. We do not detect a significant alignment of the galaxies in the filament region analyzed.
\end{abstract}

Key words. techniques: image processing - galaxies: clusters: individual: MACS J0717.5+3745 - galaxies: general techniques: photometric

\section{Introduction}

Already by the early 1980 s, Zeldovich et al. (1982) had predicted through theoretical models of structure formation that small fluctuations from the early universe would lead to a distribution of matter condensed along filaments, sheets, and voids. This resulted in the cosmic web that is now the framework for cosmology, as described for example by Bond et al. (1996). The detection of filaments is also interesting since, as underlined by Eckert et al. (2015), they could account for the missing baryons in the Universe. However, it remains difficult to detect cosmic web filaments in real data, as summarized in Libeskind et al. (2018), who compare twelve different methods to identify and classify the cosmic web. Weak lensing has been a way to detect filaments between clusters, as shown by Dietrich et al. (2012), in particular between clusters forming a pair (Planck Collaboration VIII 2013). The orientations of red galaxies have also been used as probes for filaments by Rong et al. (2016). Stoica et al. (2005) and Tempel et al. (2016) built the Bisous filament finder, a marked point process built to model multi-dimensional patterns, now publicly available. Another recent approach is the search for large-scale diffuse radio emission, as recently detected by Vacca et al. (2018), who believe this emission is linked to a large-scale filament of the cosmic web likely associated with an over-density traced by nine massive clusters.

The properties of galaxies in filaments only began to be investigated a few years ago. Based on the large-scale HORIZON-AGN hydrodynamical cosmological simulation, Dubois et al. (2014) found that at $1.2<z<1.8$ low-mass blue star-forming galaxies have a spin preferentially aligned with their neighboring filaments, while high-mass red quiescent galaxies tend to have a spin perpendicular to nearby filaments. These latter authors interpret the reorientation of galaxies as 
due to galaxy mergers, and find that the mass transition occurs around $3 \times 10^{10} M_{\odot}$. Based on simulations, comparable results were found by Ganeshaiah Veena et al. (2018), who also found that the transition mass between the two regimes increases with increasing filament diameter. Wang et al. (2018) instead found that the transition mass decreases with increasing redshift.

As far as observational data are concerned, the application of Bisous to Sloan Digital Sky Survey (SDSS) data also led to several interesting results, among which we emphasize three: First, Tempel \& Libeskind (2013) found that the minor axes of ellipticals are preferentially perpendicular to their hosting filaments. Second, Tempel et al. (2014) published a public catalog of filaments detected with Bisous, the longest filaments they detect reaching $60 \mathrm{~h}^{-1} \mathrm{Mpc}$. Third, Tempel et al. (2015) discovered a statistically significant alignment between the satellite galaxy position and the filament axis, the alignment being stronger for the reddest and brightest central and satellite galaxies.

Based on the SDSS data release 12, Chen et al. (2017) found results consistent with those based on numerical simulations: red or high-mass galaxies tend to reside closer to filaments than blue or low-mass galaxies. The star formation rate of galaxies in the outskirts of clusters was also found to be higher (Mahajan et al. 2012). Kuutma et al. (2017) investigated the impact of filament environment on galaxies, quantifying the environment as the distance to the spine of the nearest filament. These latter authors find an increase of the elliptical-to-spiral ratio while moving from voids to filament spines, but they do not detect an increase in the galaxy stellar mass while approaching filaments. They interpret their results as due to an increase in the galaxy-galaxy merger rate and/or to the cutoff of gas supplies near and inside the filaments. Their study suggests that cosmic web filaments must have an impact on galaxy properties. Indeed, Sarron et al. (2019) searched for filaments around the clusters detected by the AMASCFI software in the Canada France Hawaii Telescope Legacy Survey and showed, among other results, that preprocessing in filaments could occur in galaxy groups located in the filaments.

Galaxy luminosity functions (GLFs) can be an interesting tool to better understand the properties of galaxies in different environments. These functions have been analyzed individually for a relatively large number of clusters in a wide range of redshifts during the last few decades (Smail et al. 1998; De Lucia et al. 2004, 2007; Andreon 2006; Rudnick et al. 2009; Vulcani et al. 2011; De Propris et al. 2013; Martinet et al. 2015, 2017; Zenteno et al. 2016). Galaxy luminosity functions have also been studied in a few very large samples of clusters. These studies allowed GLFs to be stacked and their variations with cluster mass or redshift to be analyzed, separating red and blue galaxies, as recently done for example by Ricci et al. (2018) and Sarron et al. (2018). To our knowledge, GLFs have not yet been estimated in cosmic filaments, but they are expected to have properties intermediate between GLFs in dense environments such as groups or clusters, and GLFs of field galaxies. Here we discuss the properties of the GLFs in the zones covered by the filament.

In parallel, the build-up of intra-cluster light (ICL) in the current hierarchical model of evolution of big structures in the Universe is another field of interest when looking at cosmic filaments. First mentioned by Zwicky (1951) upon his discovery of an extended, low-surface-brightness, luminous halo around the Brightest Cluster Galaxy (BCG) in the Coma cluster, the ICL has been a growing field of research through recent years. Numerous studies have investigated the nature and properties of this diffuse optical component, and it is now commonly accepted that the
ICL is composed of stars that are not gravitationally bound to any cluster galaxy and are more related to the global gravitational potential of the cluster.

Intra-cluster light has been found in nearby galaxy clusters such as the Virgo cluster (Mihos et al. 2017) or the Coma cluster ( $\mathrm{Gu}$ et al. 2018; Jiménez-Teja et al. 2019) in the form of an extended luminous halo centered on the BCG, superposed on a variety of substructures such as straight streams (Mihos et al. 2005), curved arcs (Trentham \& Mobasher 1998), large plumes (Gregg \& West 1998), and tidal tails (Krick et al. 2006; Janowiecki et al. 2010). However, due to the difficulty in quantifying their morphological properties, the studies of such features remain mainly qualitative. At intermediate redshifts $(0.1<z<1)$, large samples of galaxy clusters are available, allowing systematic approaches to quantify the physical properties of the smooth ICL halo (Krick \& Bernstein 2007; Guennou et al. 2012; DeMaio et al. 2018; Jiménez-Teja et al. 2018; Montes \& Trujillo 2018; Zhang et al. 2019). These observational studies have allowed us to accumulate knowledge on the ICL under various forms, such as the fraction of ICL in galaxy clusters (from 10 to $50 \%$ ), its color, velocity, metallicity, and spatial distribution. Observations of ICL have also been made in high-redshift $(z>1)$ young galaxy clusters (Adami et al. 2013; Ko \& Jee 2018), indicating a correlation between the dynamical evolution of galaxy clusters at early times and the evolution of their ICL.

While the presence of ICL in galaxy clusters is no longer questioned, its formation mechanisms are still a matter of debate. Based on numerical simulations, Merritt (1984) showed that the ICL could form from stars dynamically stripped from their parent galaxy. Two main processes have been proposed: tidal stripping by the gravitational potential of the galaxy cluster (Byrd \& Valtonen 1990), or violent encounters between a pair or a group of galaxies, leading to the formation of large tidal streams which then mix into the ICL component (Moore et al. 1996, 1999; Mihos 2004a). Numerical works based on N-body, and hydrodynamical cosmological simulations or semi-analytic model simulations have directly investigated the effects of those formation mechanisms (Napolitano et al. 2003; Willman et al. 2004; Murante et al. 2004, 2007; Rudick et al. 2006, 2009; Contini et al. 2014, 2018). While the literature is generally consistent with the fact that a large fraction of stars is found in the ICL at $z=0$, and that the mergers and violent interactions between galaxies seem to be the main providers of those stars, there are still great discrepancies among the results, such as the time-period in which the ICL forms, its formation rate, and the relation between cluster mass and ICL fraction (Rudick et al. 2011; Tang et al. 2018).

Galaxy clusters are not the only place where diffuse light material is created. Sommer-Larsen (2006) showed in hydrodynamical simulations that galaxy groups act like smaller-scale galaxy clusters, producing their own intra-group light (IGL) through merging processes. This has been confirmed by several observational studies that found evidence for IGL in groups of galaxies (Da Rocha \& Mendes de Oliveira 2005; Aguerri et al. 2006; Da Rocha et al. 2008). One could imagine that since cosmic filaments also seem to feature a significant amount of galaxy-galaxy mergers (Kuutma et al. 2017), an extended and smooth component, the intra-filament light (IFL), could be produced in the same manner.

One way of increasing the ICL of a galaxy cluster could be through mergers with galaxy groups. This process has also been proposed by Mihos (2004b) as a potential source of ICL, as IGL could be formed in groups falling into bigger structures. 
In their work on the formation of ICL through tidal streams, Rudick et al. (2009) also showed that dynamically cold tidal streams could be formed through violent galaxy encounters in groups at early times. In some cases the tidal potential of such groups would be too weak for the streams to relax into the smooth IGL component, and the streams would remain still until their galaxy group falls into a stronger gravitational potential, to be finally mixed in the associated ICL. In the current hierarchical model of large-scale structure evolution, such groups featuring large and bright tidal streams that could become ICL in the future should be found in cosmic filaments during their infall into galaxy clusters. However, such systems have not yet been identified due to the difficulty in detecting and characterizing both cosmic filaments and ICL.

MACS J0717.5+3745 (hereafter MACS J0717) is a cluster at a redshift $z=0.5458$ known to be the most massive cluster at $z>0.5$, with a mass $M_{200}=23.6 \times 10^{14} h_{70}^{-1} M_{\odot}$ (Martinet et al. 2016). Based on HFF data, Diego et al. (2015) and Limousin et al. (2016) made a mass reconstruction of the cluster. MACS J0717 is embedded in a very long double filament of galaxies extending over more than $9 \mathrm{Mpc}$ in total (Ebeling et al. 2004; Kartaltepe et al. 2008; Jauzac et al. 2012; Durret et al. 2016). As already noted by Durret et al. (2016), neither of the two filaments (that they labeled B and C) is strongly detected in the X-rays, suggesting that we are probably dealing with cosmic web filaments linked to the cluster rather than with clusters or groups merging at large scales. This led us to choose this system to study for the first time the properties of a large-scale filament that seems to be feeding a massive cluster: the spatial distribution of the ICL, the galaxy luminosity function (GLF), which will be compared with that of the cluster itself (Martinet et al. 2017) and to field galaxy GLFs, and the orientations of the filament galaxies. The fact that MACS J0717 is covered by the Hubble Frontier Fields (Lotz et al. 2017) and therefore very deeply observed with the Hubble Space Telescope (HST) makes it an ideal object to attempt the detection of diffuse ICL with our new software, DAWIS (Detection Algorithm with Wavelets for Intra-cluster light Surveys).

The paper is organized as follows. In Sect. 3 we describe our analysis of the ICL. In Sect. 4 we present the GLF in the filament. In Sect. 5 we analyze the orientations of the galaxies in the filament. All these results are discussed in Sect. 6.

\section{The data}

\subsection{The Hubble Frontier Fields}

MACS J0717 is part of the Hubble Frontier Fields Survey $(\mathrm{HFF})^{1}$, the deepest Hubble broadband photometric survey dedicated to galaxy clusters today (ID13498, PI: J. Lotz). Longexposure images of six massive galaxy clusters in the redshift range $0.3<z<0.55$ and their parallel fields were taken in a two-step process. Epoch1 of the observing campaign for MACS J0717 took place between 2014 September and 2014 December, while Epoch2 took place between 2015 February and 2015 March (Lotz et al. 2017). The optical data were obtained with the Advanced Camera for Surveys (ACS). The IR channel of the Wide Field Camera (WFC3/IR) was used to obtain nearinfrared (NIR) images in four filters (F105W, F125W, F140W, F160W). Since the main goal of the paper is the ICL detection, we strategically chose four HST bands: F435W (UV rest frame, sensitive to star formation at the cluster redshift), F606W

\footnotetext{
https://frontierfields . org/meet-the-frontier-fields/ $\operatorname{macsj} 0717 /$
}

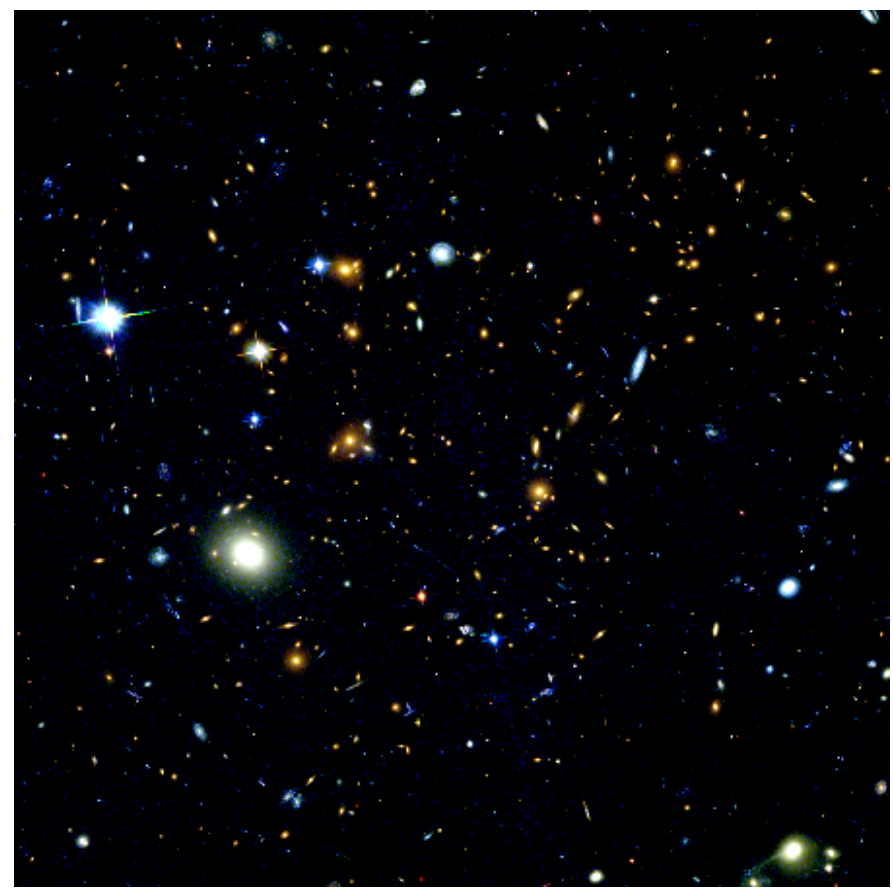

Fig. 1. Color image of the core of MACS J0717 in the Hubble Frontier Field (red: F814W, green: F606W, blue: F435W). The size of the image is $2.8 \times 2.8 \mathrm{arcmin}^{2}$ (corresponding to $\sim 1 \times 1 \mathrm{Mpc}^{2}$ at the cluster redshift).

(including the $[\mathrm{OII}] \lambda 3727$ line at the cluster redshift), F814W (the most sensitive ACS band and including the [OIII] emission lines at the cluster redshift), and F105W (including the $\mathrm{H} \alpha$ and [SII] lines at the cluster redshift). Details on the data reduction can be found in the archive handbook ${ }^{2}$.

Here we retrieve the F105W, F435W, F606W, and F814W images of the core of MACS J0717, and its parallel field from the public archive ${ }^{3}$. Two pixel sizes are available, $0.03^{\prime \prime}$ and $0.06^{\prime \prime}$. We choose the $0.06^{\prime \prime}$ pixel size to increase the sensitivity and detect low-surface-brightness objects.

The F606W and F814W filters are chosen to match the HST mosaic filters covering the filament of MACS J0717 (see Sect. 2.2), and the F435W and F105W filters are also retrieved to explore the behavior of the ICL in bluer and redder filters, respectively. Those images are used to detect ICL in the core of the MACS J0717 (see Sect. 3.2).

\subsection{Hubble Space Telescope mosaic}

The HFF (see Fig. 1) does not cover the full cosmic filament to the southeast of MACS J0717 (see Fig. 2). We therefore use another set of HST images obtained between 2005 January 9, and February 9 with the ACS (GO-10420, PI: Ebeling). This mosaic consists in 18 images of pixel size $0.05^{\prime \prime}$ in the F606W and $\mathrm{F} 814 \mathrm{~W}$ filters, and has been used in past works to detect the cosmic filament. More details can be found in Jauzac et al. (2012). This mosaic is used to compute the GLFs (Sect. 4), to study the orientations of galaxies (Sect. 5), and to look for IFL and tidal streams in the filament (Sect. 3.3). A global view of the mosaic can be seen in Fig. 2.

\footnotetext{
2 https://archive.stsci.edu/pub/hlsp/frontier/ https://archive.stsci.edu/pub/hlsp/frontier/ macs0717/images/hst/
} 


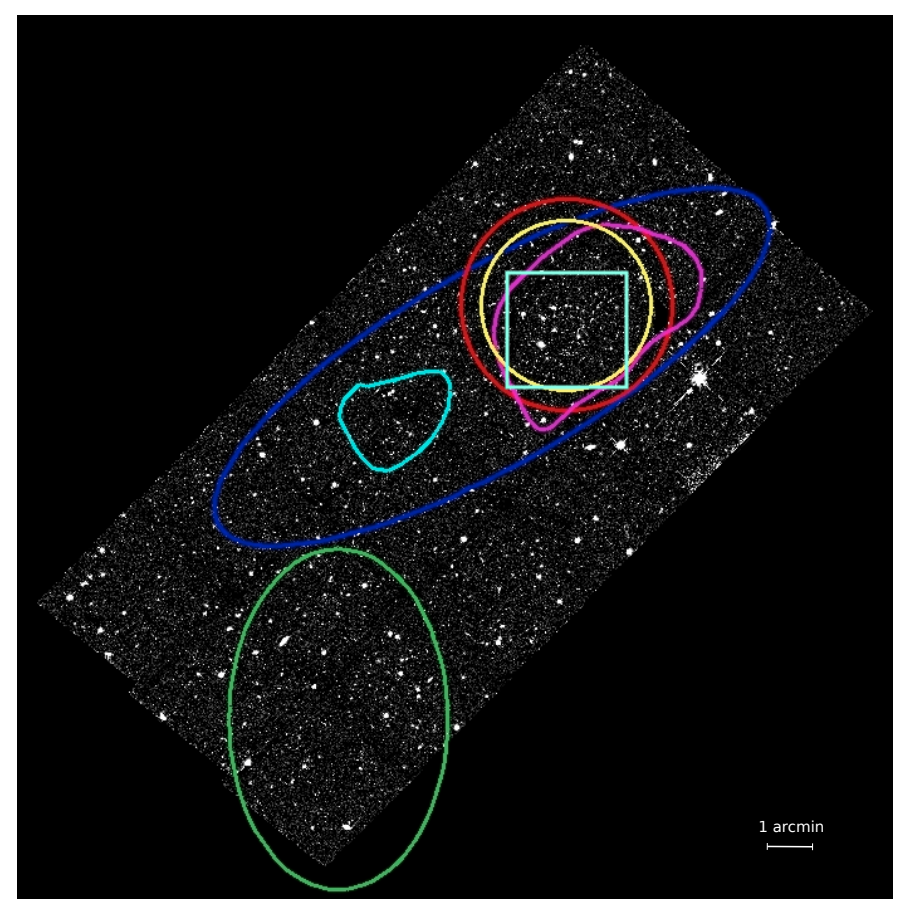

Fig. 2. Full HST mosaic, covering the entire field of MACS J0717 and its extended filament. The blue ellipse corresponds to region $\mathrm{B}$, the cosmic filament area closest to the cluster. The green ellipse corresponds to region $\mathrm{C}$, the cosmic filament area southeast of the cluster and located further. The red and yellow circles are of $1 \mathrm{Mpc}$ and $807 \mathrm{kpc}$ in radius, respectively, centered on the core of MACSJ 0717. The cyan and magenta contours show $3 \sigma$ weak lensing contours from Martinet et al. (2016). The cyan rectangle is the HFF FoV in the optical filters; see Fig. 1 for a zoom on this area.

\section{Intra-cluster light}

Here we describe our study of ICL in MACS J0717 and its filament. We first present our software in Sect. 3.1. We then apply our method to the HFF data to detect ICL in the core of MACSJ 0717 in Sect. 3.2. Finally, we describe our search for IFL under various forms in the filament of MACS J0717 in Sect. 3.3.

\subsection{Presenting DAWIS}

The ICL is a very extended and diffuse light source. Numerous instrumental factors can impact its detection, such as scattered light in telescopes, flat-fielding uncertainties, and background level estimation. The procedure to constrain these elements is given in Sect. 3.2. Other astronomical effects are also difficult to take into account, and separation of ICL from galaxy light is always a challenge. In the past decades, different methods have been applied to perform this kind of analysis: fitting and extraction of galaxy emission using light profiles (Vilchez-Gomez et al. 1994; Gonzalez et al. 2005; Jiménez-Teja \& Dupke 2016; Jiménez-Teja et al. 2018, 2019), raw masking of sources with pixel values greater than an estimated detection threshold (Burke et al. 2012; DeMaio et al. 2018; Ko \& Jee 2018; Montes \& Trujillo 2018), and using wavelet packages to model and remove galaxy light components (Adami et al. 2005, 2013; Da Rocha \& Mendes de Oliveira 2005; Da Rocha et al. 2008; Guennou et al. 2012).

While every approach has its advantages and disadvantages, the wavelet one is particularly flexible and is efficient at disentangling bright sources from low-surface-brightness diffuse ones, making it exceptionally well adapted to the detection of ICL. Indeed, contrary to fitting-oriented methods, a wavelet analysis does not need any prior information to perform detection and modeling of objects, but on the other hand its adaptability requires extended central processing unit (CPU)-time computing. With this in mind, we created DAWIS (Detection Algorithm with Wavelets for Intra-cluster light Surveys), a highly parallelized wavelet-based detection package created specially for the detection and study of ICL. DAWIS is optimized to run on large images faster than regular linear wavelet packages, in order to process large amounts of data.

In the following sections, we give a global description of DAWIS, which consists in the wavelet convolution of an astronomical image, the detection of objects in wavelet space, and the reconstruction of these detected objects. More detailed explanations and tests on simulations can be found in Ellien et al. (in prep.).

\subsubsection{Wavelet convolution}

Astronomical images can be hierarchically decomposed: bright compact sources such as stars or galaxy cores are located in larger envelopes like star halos or galaxy disks, which are themselves enclosed in very large, diffuse low-surface-brightness sources such as the ICL. All these objects are projected on the sky background, the largest component (covering the entire image) setting the surface brightness detection limit. A multiscale approach is ideal to disentangle these different luminosity scales and to study them.

DAWIS is based on Mallat's à trous wavelet algorithm (Shensa 1992), which is particularly suited for astronomical images. It is a fast, discrete, redundant wavelet transform that respects flux conservation. Going through this algorithm, an image is convolved in $N_{\text {lvl }}$ wavelet planes, following a multiscale vision as described in Bijaoui \& Rué (1995). The process is done by iteratively smoothing the original image $N_{\mathrm{lvl}}+1$ times with a varying B-spline kernel, the difference between two successive smoothed images giving a wavelet plane. Each wavelet plane contains features with a characteristic size of $2^{n}$ pixels, $n$ being the index of the plane $\left(n=0,1,2,3, \ldots, N_{\text {lvl }}\right)$. Small values of $n$ correspond to bright and compact luminous features, while greater values correspond to large and low-intensity ones, with $n=N_{\mathrm{lvl}}$ corresponding to the large sky background variations. The maximal number of wavelet planes $N_{\text {lvl,max }}$ that can be obtained for one image is given by its size which is $2^{N_{\text {lvl,max }}}$ pixels.

\subsubsection{Detection of objects}

After a wavelet convolution, astronomical objects are decomposed into several features through the different wavelet planes. In each plane, we apply a thresholding to determine the statistically significant pixels composing these features. Noise representation in wavelet space is an important attribute of this approach. Indeed, the result of a wavelet convolution of Gaussian noise is also Gaussian noise, but with a shift in intensity depending on $n$. Astronomical noise is strongly dominated by small characteristic size variations, and the first wavelet plane noise $(n=0)$ is for the most part high-intensity pixel-topixel noise. The noise intensity falls drastically with increasing $n$, revealing astronomical objects. This means that in large $n$ wavelet planes, extended and low-surface-brightness features are easily identified because of greatly reduced background noise. 
Table 1. Detection threshold and ICL fractions computed for each of the four filters of the HFF.

\begin{tabular}{lcccc}
\hline \hline HFF & F435W & F606W & F814W & F105W \\
\hline $3 \sigma_{\text {bkg }}\left(\mathrm{mag} \mathrm{arcsec}^{-2}\right)$ & 29.89 & 29.96 & 30.03 & 29.97 \\
$5 \sigma_{\text {bkg }}\left(\mathrm{mag} \mathrm{arcsec}^{-2}\right)$ & 29.34 & 29.41 & 29.50 & 29.41 \\
Radius $(\mathrm{kpc})$ & 275.3 & 562.5 & 421.5 & FoV \\
$f_{\text {ICL }}(\%)$ & $2.48_{-0.20}^{+0.19}$ & $24.43_{-1.71}^{+3.37}$ & $16.10_{-1.03}^{+1.03}$ & $13.22_{-1.49}^{+1.76}$ \\
\hline
\end{tabular}

Notes. The thresholds are used to create the ICL maps from the residual images (see Fig. 5). The fractions are computed from the ICL maps and the reconstructed images in the four filters (see Sect. 3.2.4) within the radius that is indicated for each filter. Error bars correspond to the 95\% confidence interval computed from bootstrap resampling (see text for details). The radii are the same as in Jiménez-Teja et al. (2019) for comparison purposes.

The detection threshold is estimated in each wavelet plane separately: a standard deviation of the intensity, $\sigma_{n}$, is computed using a $3 \sigma$ clipping algorithm, and the significant pixels are set to have intensity values higher than $k \sigma_{n}$. This threshold is different for each wavelet plane, but the same value $k$ is applied everywhere, and we refer from now on to these different thresholds as $k \sigma_{\mathrm{w}}$, the detection threshold in the wavelet space (usually $3 \sigma_{\mathrm{w}}$ or $\left.5 \sigma_{\mathrm{w}}\right)$.

After thresholding, the significant pixels are grouped in regions using scale-by-scale segmentation. We then create interscale trees by linking together significant regions from different planes by looking at their spatial distribution. Trees with connected regions from at least three different planes are recognized as valid representations of astronomical objects in the wavelet space.

\subsubsection{Reconstruction of objects}

For each detected inter-scale tree, the region with the highest pixel value is set as the main region. The information from this region, and every region of the tree that belongs to smaller $n$ planes, is used to reconstruct the object in real space, using a conjugate gradient algorithm (Starck et al. 1998). All the reconstructed objects are then inserted in a single image that we call the "reconstructed image". A residual image is produced by subtracting the reconstructed image from the original astronomical one. We refer from now on to the full process of wavelet convolution, detection in wavelet space, reconstruction of every detected object, and computation of reconstructed and residual images as a "run".

The fact that only information from the main region and regions with smaller indexes is used to reconstruct an object means that the priority of DAWIS is to detect and reconstruct bright and compact sources. Many low-surface-brightness sources can be missed in this way and are found in the residual image. In that case, a second DAWIS run can be applied to this residual image. Objects that have not been detected in the first run are then detected, reconstructed, and subtracted from the first residual image, producing a second residual image. This iterative process can be generalized to $N$ runs if needed. For example, the number of runs can be pushed to eight to create masks (see Sect. 3.2.3).

\subsection{Intra-cluster light in the core of MACS J0717}

Here we describe the data processing applied to the HFF images in order to quantify the contribution of ICL in the core of MACS J0717 to the total luminosity budget.

\subsubsection{Background estimation}

An accurate estimation of the sky background is essential to the study of low-surface-brightness features, as the subtraction of the nonuniform background could erase significant signal. The task is particularly complicated if those features are extended over large areas, since a classical global estimation of the sky background is contaminated by the light of diffuse sources. In the case of the HFF images, the field of view (FoV) is smaller than the actual size of MACS J0717 and the ICL might cover a large portion of it (potentially the full image). More sophisticated methods of background estimation are then necessary (DeMaio et al. 2018; Ko \& Jee 2018; Montes \& Trujillo 2018).

For the HFF data, we take advantage of the deep parallel fields available (Lotz et al. 2017), and use them to compute the sky background. The HFF parallel fields are pointed at a single target distant by $\sim 6$ arcmin from the galaxy cluster core (equivalent to $\sim 2.2 \mathrm{Mpc}$ at the redshift of MACS J0717), in a region covering the galaxy field. This is far enough from the cluster center to avoid large ICL contribution, and should provide a fair estimation of the sky background.

In the HFF images, the subtraction of a constant sky background was performed during the data reduction. In order to get rid of negative pixel values which DAWIS cannot deal with, we add a constant to every image we process during this work, and remove it from the output images at the end of the wavelet processing. For each band we create $\sim 50$ random circular regions of radius $3.6^{\prime \prime}$, covering the entire parallel field. This size is chosen to be larger than typical field galaxies, but small enough to avoid large-scale background variations. For each region, we use a $3 \sigma$ clipping algorithm to estimate a standard deviation of the pixel values, which allows us to remove the bright sources that can be found above the average intensity level in some of them. Subsequently, we once again apply a $3 \sigma$ clipping algorithm, but this time on the values of the standard deviation for all regions, removing outliers. The final value obtained is the global standard deviation $\sigma_{\mathrm{bkg}}$. We insist here on the fact that $\sigma_{\mathrm{bkg}}$ is different from $\sigma_{\mathrm{w}}$. The latter is computed in the wavelet space and used to detect objects before they are reconstructed (see Sect. 3.1.2), while $\sigma_{\mathrm{bkg}}$ is used to compute the detection threshold in the final residual map after all of the wavelet processes are done. The $3 \sigma_{\mathrm{bkg}}$ and $5 \sigma_{\mathrm{bkg}}$ detection thresholds for each filter are given in Table 1.

\subsubsection{Point spread function}

The point spread function (PSF) is the generic term used to characterize the response of an instrument, telescope, or detector to a point-like source, and is the combination of a variety of effects such as the diffraction pattern induced by the telescope aperture, optical aberrations, scattered light within the instrument, and atmospheric turbulence, resulting in a blurring of the source image. While we observe this deformation, the variety of these 
effects and their different origins make it difficult to estimate or model them accurately, and each instrument requires specific study and characterization of its PSF.

We measure the PSF on bright nonsaturated stars. While the inner part is by far the most luminous one, the wings are also important. These wings can be a source of contamination for low-surface-brightness features (Sandin 2014, 2015), bringing light from the inner parts of galaxies to their outer halo and modifying their color properties and luminosity profile at large radii (Capaccioli \& de Vaucouleurs 1983; de Jong 2008; Trujillo \& Fliri 2016). This effect also impacts the ICL, since light from galaxies can be artificially brought to the intergalactic medium, simulating flux emitted by diffuse low-surfacebrightness sources and polluting real ICL.

Correcting an astronomical image for PSF effects is not an easy task, as PSF properties depend on wavelength, spatial position on the image, and exposure time, among others. Consequently, the PSF of a science image is usually measured empirically, with tools like PSFEx (Bertin 2011, 2013). This method estimates the PSF directly from stars in the science image by stacking them, and can measure the PSF accurately up to radial distances of a few arcseconds. Other stacking methods to increase the signal-to-noise ratio of the PSF wings have been used in some works (Janowiecki et al. 2010; Karabal et al. 2017; DeMaio et al. 2018), but require numerous stars and a specific observation strategy to avoid saturation.

In our case, the small FoV of the HFF images strongly reduces the number of stars available for PSF measurements. For this reason, we choose to use TinyTim, a modeling tool that has been created to provide PSFs for all the instruments and observation modes of the HST (Krist et al. 2011). TinyTim takes into account many factors for the making of the PSF, such as aberrations, time-dependent focus, and geometric distortions (more details are available in the TinyTim User Guide available on the project website ${ }^{4}$ ).

We create a master PSF for each of the F435W, F606W, F814W, and F105W filters of the HFF. Since the PSF size is not the same for each filter, we homogenize the process and create PSFs within a radius $r \sim 19.5^{\prime \prime}$. This is the maximum possible size for F435W with TinyTim, and is the smallest of all four filters. The PSFs are then rotated to match the camera angle, and re-sampled to the pixel size of the HFF images $\left(0.06^{\prime \prime}\right)$. The images are then deconvolved from the PSF with a RichardsonLucy algorithm (Richardson 1972; Lucy 1974).

\subsubsection{Masking and wavelet processing}

Bright foreground stars are a major problem as DAWIS cannot reconstruct them properly, making the masking of these objects mandatory before applying any wavelet process. Otherwise, the strong signal of these objects is found everywhere in the wavelet convolution, contaminating every scale and preventing any type of reliable detection and reconstruction of objects. While the PSF deconvolution helps in this instance by removing most of the bright components, a few obvious star residuals remain, due to the fact that we did not take into account every variable while computing the PSF with TinyTim, such as spatial variation on the image.

Here the meaning of the term "mask" is slightly ambiguous, as we do not simply set values covered by the mask either to zero or to $\mathrm{NaN}$ values. Indeed, the wavelet convolution requires a value for each pixel of the image (prohibiting the use of $\mathrm{NaN}$

\footnotetext{
4 http://www.stsci.edu/hst/observatory/focus/TinyTim
}

values), and large regions of zero values would interact strangely with other features of the image, creating artifacts and ghost objects. Since wavelets act as filters in the Fourier space, we replace the values of the masked pixels by random Gaussian noise values using $\sigma_{\mathrm{bkg}}$, and the same mean value of standard deviation that we computed in Sect. 3.2.1. The underlying idea is that a mask for a wavelet convolution is a region for which there must be zero correlation with the rest of the image, which is by definition Gaussian noise.

Other sources that sometimes bring additional complications are very large foreground elliptical galaxies. The cores of those objects are several orders of magnitude brighter than their halos, making the two components hard to recognize as a single object from the wavelet perspective. For elliptical galaxies at the cluster redshift, the tricky part is to determine at what point the light stops belonging to the galaxy, and starts becoming ICL, but the wavelet convolution is very efficient in decoupling the two components, as they belong to two different luminosity scales. However, for foreground galaxies, this becomes a problem if the luminosity of such a halo merges perfectly with the ICL, both covering the same ranges of surface brightness as a result of the foreground galaxy being closer to us. In such cases, it is almost impossible to determine the real origin of the light at large radii, and DAWIS cannot differentiate the external parts of this halo from ICL.

In the case of MACS J0717, a very large and bright foreground galaxy is situated close to the BCG in the projected sky plane (see Fig. 1, the large yellow elliptical galaxy in the bottom left corner), and the light emitted by this galaxy occupies a very large part of the image. Here we dedicate a specific wavelet treatment to this object. We extract a patch around the galaxy enclosing the full halo and its low-surface-brightness parts, and run DAWIS in a mode where we only reconstruct detected objects that are centered on the galaxy itself. The idea is to model the galaxy light profile down to very low surface brightness (ICLlike scales), and to remove it from the original FF image, before applying DAWIS to the whole field. We push the number of wavelet detections, reconstructions, and subtractions up to $\sim 5-8$ consecutive runs (depending on the filter), in order to precisely model and remove as much light as possible, and obtain a map of residuals of mean and standard deviation comparable to the background computed in Sect. 3.2.1.

There are two downsides to this method. The first one is that the external parts of the luminosity profile detected and modeled by DAWIS for this galaxy could be amplified by ICL belonging to MACS J0717. However, we prefer to slightly over-subtract the ICL of MACS J0717 than to have this strong source of contamination in the results of our study. The second downside is that the extraction of the profile is time-consuming, considering we are running DAWIS several times for a single galaxy, and therefore such a treatment should be restricted to critical cases such as this one.

Once all problematic objects or residuals have been masked or removed, the core wavelet processing of the whole field can start. In order to save some computer time, we re-sample every image to a $0.24^{\prime \prime}$ per pixel scale, which is a flux conservative process. We then apply the following wavelet process to the four filters (F435W, F606W, F814W, and F105W) of the HFF.

1. A first run of DAWIS with a very high wavelet detection threshold $(k=10)$ and a small convolution kernel $\left(N_{\mathrm{lvl}}=6\right.$, corresponding to a characteristic object size of 64 pixels), in order to detect bulges and other very bright and localized sources. The wavelet planes of the F814W image can be found in Fig. 3 as an example illustrating this step. 


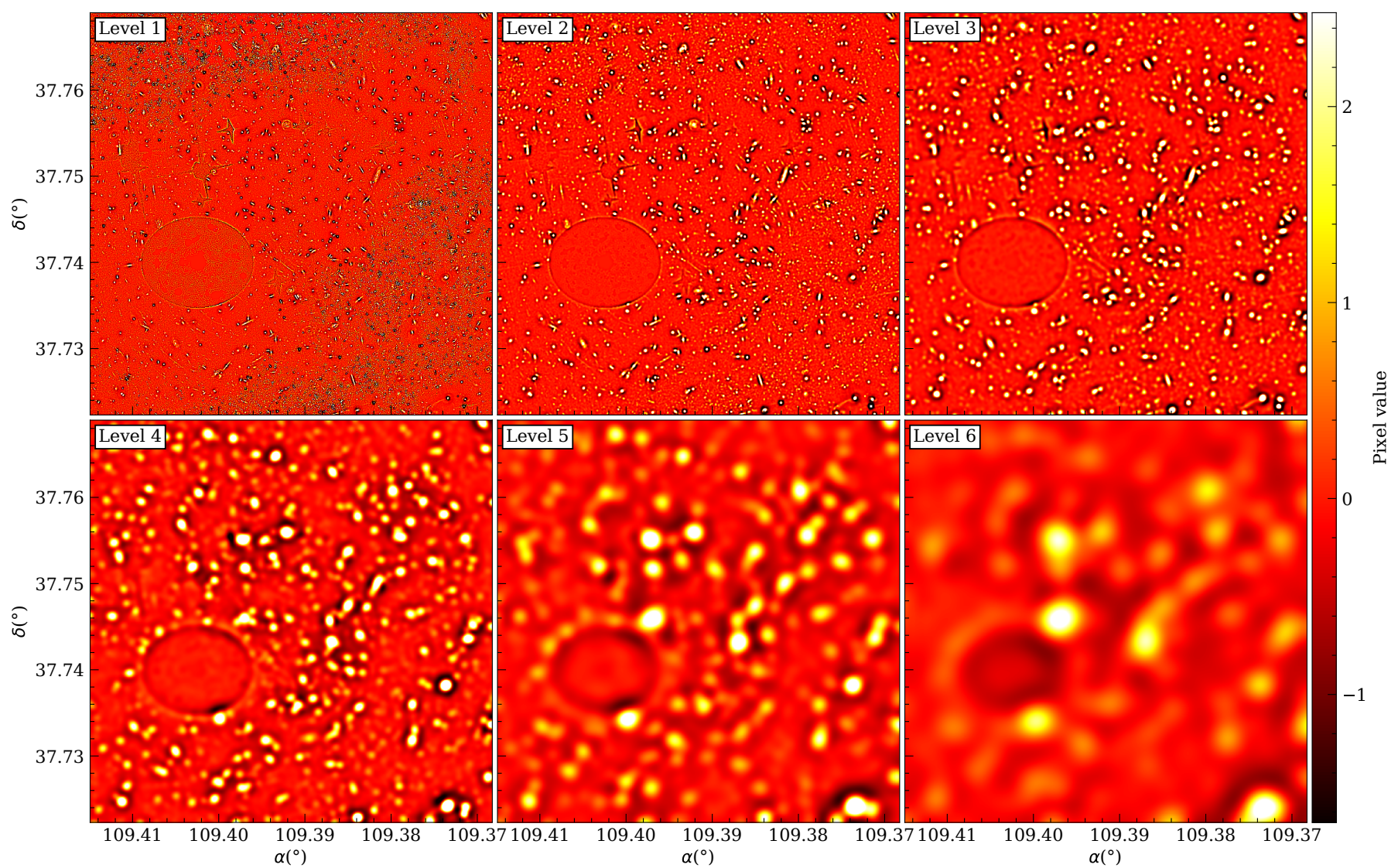

Fig. 3. Wavelet planes of the first run of DAWIS on the F814W image. There are six planes, corresponding to maximum characteristic object sizes of 64 pixels. As the level of the plane increases, the size of sources becomes larger and their intensity decreases. When the thresholding is done, the negative coefficients are simply ignored.

2. A second run of DAWIS with a lower wavelet detection threshold $(k=5)$ and a larger kernel $\left(N_{\mathrm{lvl}}=7\right.$, corresponding to a characteristic object size of 128 pixels), in order to detect disks and outer halos of galaxies.

3. A third run with an even lower detection threshold $(k=3)$ and with $N_{\mathrm{lvl}}=9$ (the maximum value given the size of the images), which gives a characteristic object size of 512 pixels. Extended halos that have been missed in the second run can be detected this way.

A complete reconstructed image of every object detected by DAWIS can be created by stacking the reconstructed images of the three runs (see Fig. 4). There is excellent agreement between the original and reconstructed images, which demonstrates the ability of DAWIS to separate compact objects (such as galaxies) from more extended sources (the ICL) that remain in the residual image.

\subsubsection{Results}

To create the ICL maps in the HFF, we choose to use the residual images after the three DAWIS runs. For each filter, we attribute pixels in the residual maps with a value above $3 \sigma_{\text {bkg }}$ to the ICL ( $\sigma_{\mathrm{bkg}}$ is the global standard deviation of the sky background computed in Sect. 3.2.1). The final ICL maps are given in Fig. 5, showing $3 \sigma_{\mathrm{bkg}}$ and $5 \sigma_{\mathrm{bkg}}$ contours.

The spatial distribution on the projected sky of the ICL in each filter is consistent with previous works on MACS J0717 such as in Morishita et al. (2017, see their Fig. 2), or in Montes \& Trujillo (2019, see their Fig. 3). The fact that the morphology of the ICL differs from one filter to another is interesting, as it indicates the presence of different populations of stars in the ICL.

We retrieve galaxy spectroscopic redshifts from the NASA Extragalactic Database ${ }^{5}$ (NED) and select only galaxies in the FoV of the HFF and with $0.53<z_{\text {spec }}<0.56$, assuming galaxies in this range of redshift are associated with MACS J0717. We add the position of the spectroscopically confirmed cluster member galaxies on the residual maps. We note that while this galaxy catalog is a good representation, it is far from being complete. Instead, we use the red sequence (RS) computed in Sect. 4 to compute ICL fractions in each band:

$f_{\mathrm{ICL}}=\frac{F_{\mathrm{ICL}}}{F_{\mathrm{gal}}+F_{\mathrm{ICL}}}$,

where $F_{\text {ICL }}$ is the integrated flux of ICL and $F_{\text {gal }}$ is the integrated flux of the galaxies belonging to MACS J0717. $F_{\text {ICL }}$ is obtained by summing the pixel values with values greater than $3 \sigma_{\text {bkg }}$ in the ICL maps, and $F_{\text {gal }}$ is obtained by summing the pixel values of the RS galaxy profiles in the reconstructed images. In order to compare our approach with recent works, we measure the ICL fractions in the same radii centered on the BCG as in Jiménez-Teja et al. (2019). The computed ICL fractions can be found in Table 1.

The errors on the ICL fraction are computed with a bootstrap on the values of the pixels of the galaxies and of the pixels of the ICL. For each filter, we create a sample with all the pixels belonging to RS galaxies of the reconstructed image, and a

https://ned.ipac.caltech.edu/ 

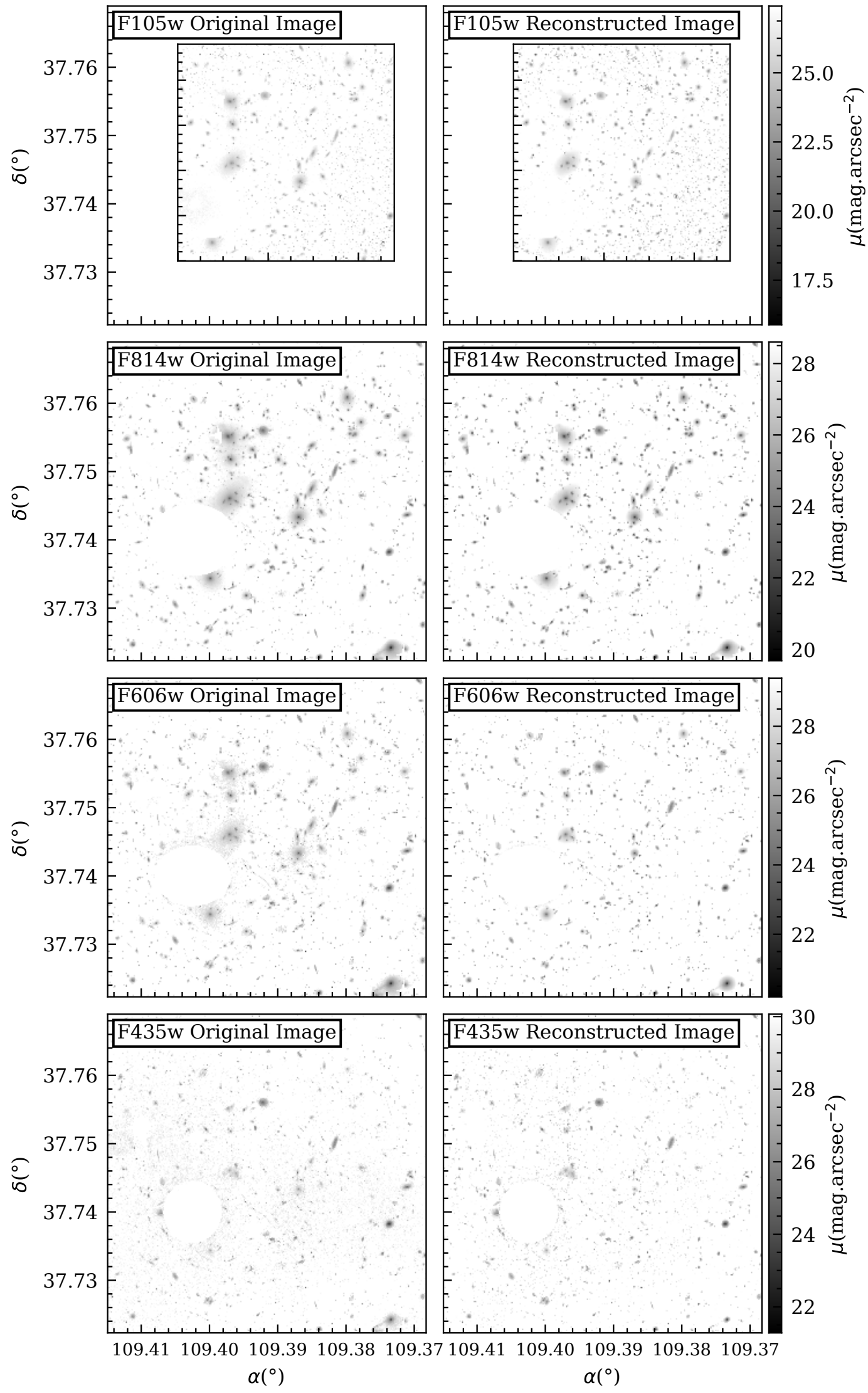

Fig. 4. Left column: original HFF images in the four filters after PSF deconvolution and masking of star residuals and of the large foreground galaxy. Right column: stacked images of objects detected and reconstructed by three runs of DAWIS. The F105W residual map is smaller as a result of the WFC3 FoV being smaller than the ACS one, and has therefore been scaled to the other ones. 


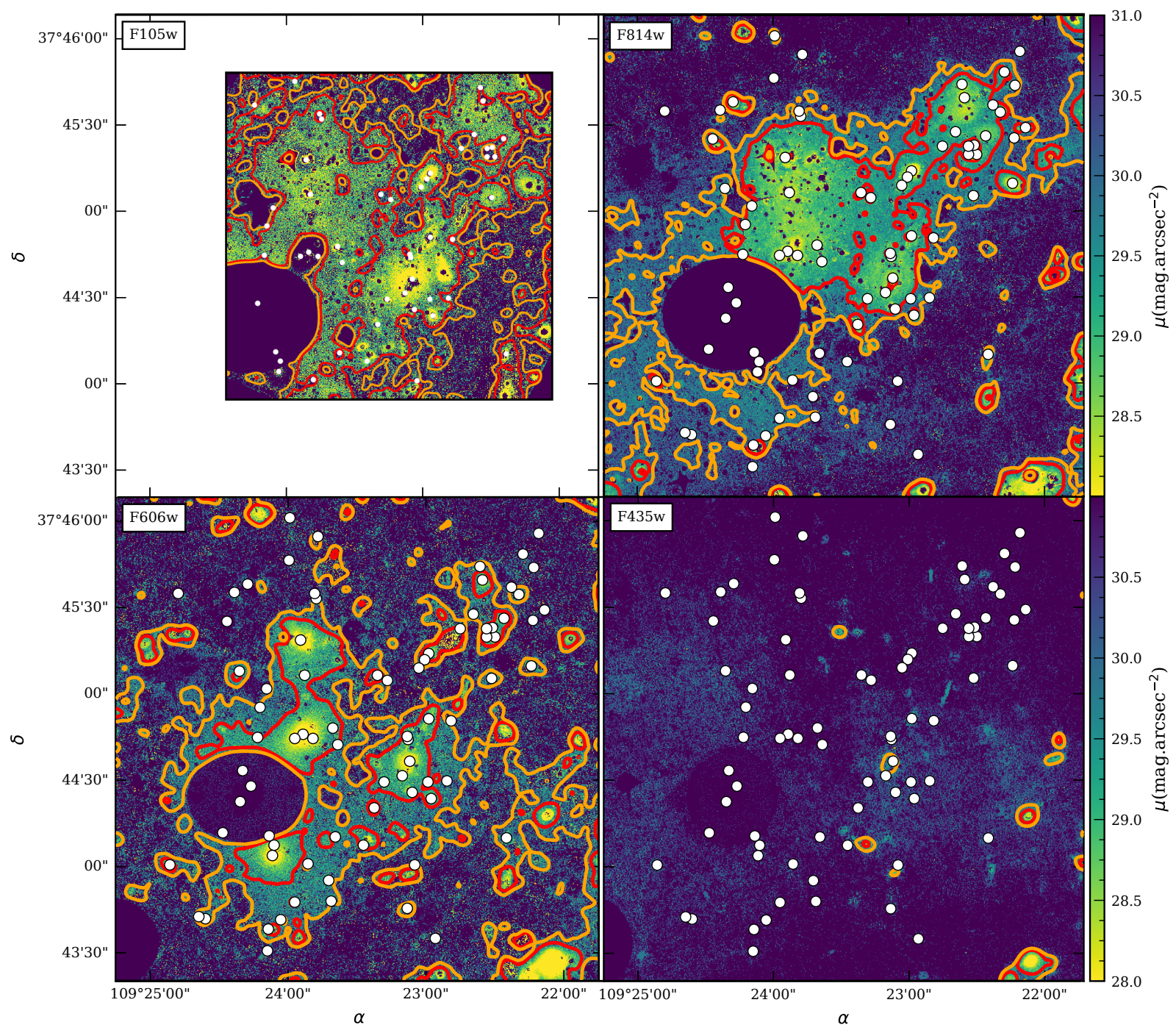

Fig. 5. Surface brightness maps of the residuals after wavelet processing by DAWIS in each band. The orange contours show $3 \sigma_{\text {bkg }}$ detection and the red ones $5 \sigma_{\mathrm{bkg}}$. The white dots show the galaxies in the cluster redshift range $(0.53<z<0.56)$. From top to bottom and left to right: $\mathrm{F} 435 \mathrm{~W}$, F606W, F814W, and F105W. The F105W residual map is smaller as a result of the WFC3 FoV being smaller than the ACS one, and has therefore been scaled to the other ones. The contours are smoothed with a Gaussian kernel of $\sigma=5$ for the map to be readable.

sample with all the ICL pixels of the residual image. We draw $N=10000$ sub-samples randomly from each sample, allowing the same pixel value to be drawn multiple times. We then compute the given ICL fraction for each sub-sample which gives $N$ values of ICL fractions for each filter. The errors on the true ICL fraction value are then estimated by computing a $95 \%$ confidence interval on the sub-sample values.

We find ICL fractions in good agreement with the ones in Jiménez-Teja et al. (2019) in the F606W and F814W filters. Our fraction in the F435W filter differs significantly (we find a value of $2.5 \%$ compared to their value of $7.22 \%$ ), which could be due to a number of differences in the data processing, since the sky level is computed differently in both studies, or to the fact that these authors used a different analysis package CICLE (CHEFs Intracluster Light Estimator; Jiménez-Teja \& Dupke 2016) to extract and remove galaxy light profiles. In the case of F105W (not analyzed by Jiménez-Teja et al. 2019), we simply integrated the whole FoV to compute the fraction. We find that the fraction of ICL is peaking in the blue F606W filter before decreasing progressively in the $\mathrm{F} 814 \mathrm{~W}$ and $\mathrm{F} 105 \mathrm{~W}$ filters as it gets redder.

\subsection{Detection of IFL in the filament}

In this section, we look for IFL in the regions corresponding to the filament of MACS J0717 (regions B and C; see Fig. 2 for a global view of the system). We first investigate whether or not we detect a global diffuse component in the filament, as in the core of MACS J0717, then we look at perturbed systems of galaxies in the filament presenting evident tidal streams.

\subsubsection{Detection of diffuse sources}

Since the HST mosaic is not as deep as the HFF, we test the feasibility of the detection of ICL in those images. For this, we try to re-detect in the corresponding HST images the ICL previously found in the core of MACS J0717 (see Sect. 3.2). After binning the image from $0.05^{\prime \prime}$ to $0.24^{\prime \prime}$ per pixel, the stars and the foreground galaxy are masked by hand. We do not deconvolve the image by the PSF, since the result is not used in any way other than testing the detection limit of the mosaic. Contrary to the HFF, there is no parallel field to compute the sky background level, but since we now know the spatial 


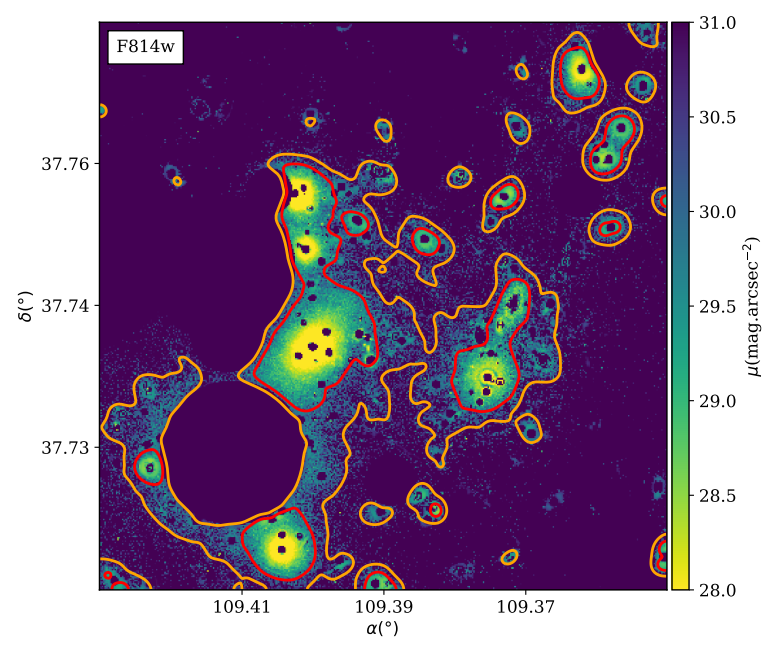

Fig. 6. Close-up on the ICL in the core of MACS J0717 in the HST mosaic F814W image. The stars and the large foreground galaxy are masked by hand (the external halo of the galaxy is still visible here), and the same wavelet processing as for the HFF has been applied. The contours show the $3 \sigma_{\mathrm{bkg}}$ detection limit. Part of the ICL contribution is detected in this image, showcasing the fact that this mosaic is deep enough to detect the brightest component of the ICL.

distribution of the ICL, we use the same method as in Sect. 3.2.1. This time however, the regions are created directly in the image used for the detection, avoiding the areas where ICL is detected in the HFF. We then run exactly the same wavelet process as in Sect. 3.2.3 on the whole image. This gives a $3 \sigma_{\mathrm{bkg}}$ detection threshold of $28.54 \mathrm{mag} \mathrm{arcsec}^{-2}$ for the F814W image, and $27.59 \mathrm{mag} \mathrm{arcsec}^{-2}$ for the F606W image.

The test is not conclusive in the case of the F606W image which is too shallow to reach the surface brightness level necessary to detect ICL, and as the residual image shows many factors of contamination due to flat-fielding uncertainties. In the F814W residual image however, we are able to detect once again ICL in the core of MACS J0717 (see Fig. 6), meaning that the mosaic in the F814W filter is deep enough to detect large sources of equivalent surface brightness that could be associated to IFL in the filament. We apply the same process to every image of the HST mosaic covering parts of the filament in the F814W filter. If possible, the background regions are created in areas outside of the contours demarcating regions $\mathrm{B}$ and $\mathrm{C}$ shown in Fig. 2. If not, they are created at random positions in the image.

We do not detect any significant source of light that could be associated to IFL, as the residual maps mostly contain noise, flatfield uncertainties, and wavelet residuals. This is not really surprising, as strong diffuse light features are associated to galaxy clusters or groups (respectively ICL and IGL) and are believed to be formed through many galaxy-galaxy gravitational interactions such as mergers. This absence of IFL detection seems to confirm this formation scenario, as cosmic filaments do not undergo as many gravitational interactions as galaxy groups or clusters (in our case, interactions are not sufficient to create an amount of IFL equivalent to the amount of ICL in the core of MACS J0717).

\subsubsection{Detection of tidal streams}

In parallel, we looked for tidal streams in the cosmic filament of MACS J0717, as simulations have shown that significant portions of ICL could be formed in such structures (Rudick et al.
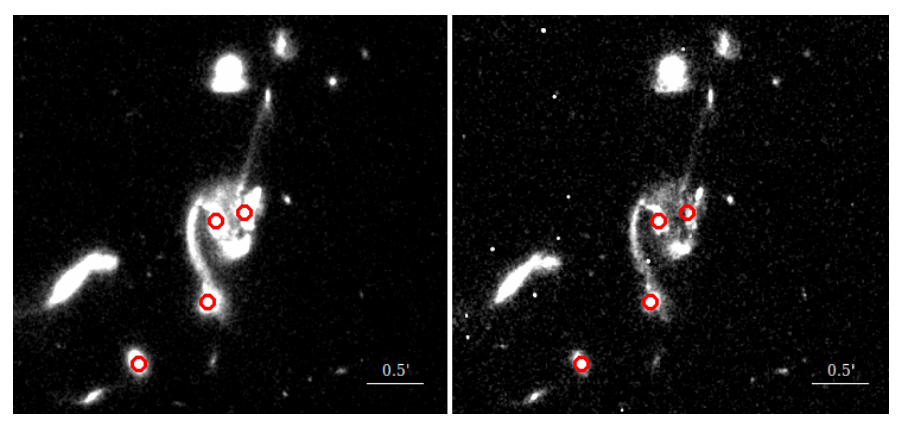

Fig. 7. Close-up on the disturbed system of galaxies in region B of the cosmic filament. Left: F814W filter. Right: F606W filter. The red circles mark galaxies with spectroscopic redshifts in the range $[0.53,0.56]$.

2009). The detection of tidal streams is a tricky task due to their various morphologies, and while some could be found in the DAWIS residual images, we cannot fully differentiate them from wavelet residuals and artifacts. We choose instead to detect the presence of candidate tidal streams by visually inspecting every image of the HST mosaic. The work in this section is only qualitative and is meant to be a complement to the study of the ICL in the core of MACS J0717, and a pinpoint for future studies.

We look first at every galaxy with a spectroscopic redshift in the range $[0.53,0.56]$, then at every galaxy of the RS computed in Sect. 4 . The criteria used to determine the tidal stream candidates are the following: (i) presence of several galaxies with close positions on the projected sky plane; (ii) signs of very disturbed galaxy morphology; (iii) presence of low-surface-brightness features such as tidal streams or arcs associated with these galaxies. Several inspections were independently done by different persons of our group before comparing the results and picking the candidates.

In the whole mosaic we find only one system matching these criteria, in region $\mathrm{B}$ of the filament. This system is composed of three interacting galaxies with spectroscopic redshifts, presenting obvious signs of tidal stripping, such as a linear stream, an arc, and a diffuse envelope around the core of the galaxies (see Fig. 7). This system resides within the weak lensing contours of Martinet et al. (2016, see Fig. 2), indicating that it belongs to a massive substructure, such as a galaxy group embedded in the cosmic filament (see also Sects. 4 and 5), and producing its own IGL through tidal streams. The fact that the only system of galaxies presenting obvious tidal streams in the whole cosmic filament seems to actually belong to this galaxy group suggests that the creation of IFL through galaxy-galaxy mergers directly in the filament (e.g., outside of a massive sub-structure) is not possible.

\section{Galaxy luminosity functions}

This section aims at better understanding the distribution of galaxy luminosities within filaments. This is done by measuring the GLFs in the structures surrounding MACS J0717 and comparing them with the cluster and field GLFs.

\subsection{Computing galaxy luminosity functions}

The building of the GLFs follows that of Martinet et al. (2017) and we refer the reader to this paper for a detailed description of each step. Here we only recall the salient points of the analysis and those which differ from the mentioned study.

The detection of objects is made individually on each image of the HST mosaic with the SExtractor software 
(Bertin \& Arnouts 1996), and the catalogs are then concatenated in a single catalog for each band. Because the cluster core and the mosaic covering the filament were observed at different epochs and with different observing strategies, the astrometry between the two sets of images does not match with a sufficient accuracy. This results in some objects in the overlapping area between these images being detected several times. For each of these objects we discard the detection with the lowest signal-to-noise ratio, applying a matching of objects in separation, magnitude, and surface brightness in the mentioned area. Due to the same astrometric issue, the detection is made independently in the F814W and F606W bands, contrary to Martinet et al. (2017) where we used the double image mode of SExtractor to measure the flux in the F606W filter in the same apertures as those detected in the F814W filter. In the present study, the two catalogs are then cross-matched in a closest-neighbor approach, with a maximum separation of 2 arcsec. We also discard spurious or contaminated detections using hand-made masks around bright saturated stars and on noisy image edges. All the magnitudes discussed hereafter correspond to SExtractor MAG_AUTO measurements.

Galaxies are then separated from stars based on a maximum surface brightness versus magnitude diagram, in the F814W filter up to magnitude 25 .

We select RS galaxies in a color magnitude diagram through an iterative process. We first consider a broad RS centered on the F814W-F606W color of elliptical galaxies at the cluster redshift applying prescriptions from Fukugita et al. (1995). The RS is then refined with a linear fit to the selected galaxies by fixing the slope to -0.0436 , which has been shown to be a constant value for clusters in this redshift range (Durret et al. 2011). The final width of the RS is set to \pm 0.3 in color.

We subtract field galaxies in apparent magnitude after applying the same RS color cut as that of the cluster galaxies and normalizing both cluster and field galaxy counts to $1 \mathrm{deg}^{2}$. The sample of field galaxies we used is that of Martinet et al. (2017), which corresponds to a subarea of $\sim 0.05 \mathrm{deg}^{2}$ of the COSMOS survey $^{6}$ re-analyzed by the 3D-HST team (Brammer et al. 2012; Skelton et al. 2014), and for which we applied the same detection setup as in the present study.

We compute restframe absolute magnitudes for RS galaxies by applying the distance modulus at the cluster redshift and a constant $k$-correction for all galaxies. This assumes that RS galaxies lie at the same redshift and have identical colors, which is a generally good approximation for RS galaxies (see e.g., Martinet et al. 2017). The $k$-correction is computed as the mean over a series of early-type spectral energy distribution templates in a \pm 0.05 redshift range around the cluster redshift using the LePhare software (Arnouts et al. 1999; Ilbert et al. 2006).

The last steps assume that galaxies in the filaments also lie in the cluster RS, an assumption that is discussed below when interpreting the results. We note that in the present study it is not possible to consider photometric redshifts, since we only have two optical bands in the region covered by the filament.

Each magnitude bin is assigned an error bar which corresponds to the quadratic sum of the Poisson errors on the field, and on the cluster or filament counts. Given the very deep images of MACS J0717, the completeness limit is set by the depth of the COSMOS field galaxies, determined from their magnitude histogram. These limits correspond to a magnitude of 26 in both F814W and F606W filters. GLFs:

Finally, we fit Schechter functions (Schechter 1976) to the

6 http://cosmos.astro.caltech.edu/
$N(M)=0.4 \ln (10) \phi^{*}\left[10^{0.4\left(M^{*}-M\right)}\right]^{(\alpha+1)} \exp \left(-10^{0.4\left(M^{*}-M\right)}\right)$,

constraining the three following parameters: the slope of the faint end $\alpha$, the characteristic magnitude of the bending $M^{*}$, and the normalization $\phi^{*}$. The fit is performed via a $\chi^{2}$ minimization.

\subsection{Red sequence galaxy luminosity functions}

We compute the GLFs in six different areas of the HST mosaic. First, we study the GLF in a region within a $807 \mathrm{kpc}$ radius from the cluster center. This is the same area as the one studied in Martinet et al. (2017) for the same cluster with HST data, and we study it here to verify that our analysis is consistent with previous ones. Second, we measure the GLFs in the $3 \sigma$ weak lensing (WL) contours shown in Fig. 2 (as in Martinet et al. 2016). These contours correspond to the total overdensity, both luminous and dark matter, as probed by the lensing of background sources by the gravitational potential of the foreground structures. Martinet et al. (2016) reported an almost $11 \sigma$ detection of the cluster itself and a $\sim 8 \sigma$ detection of a filamentary structure to the southeast from Subaru/Suprime-Cam images, which was also detected in the WL analysis of the HST mosaic used in the present paper (Jauzac et al. 2012). Finally, we compute the GLFs in a $1 \mathrm{Mpc}$ radius centered on the cluster, and in two ellipses defined from the overdensities of luminous RS galaxies after filtering the galaxy density field with a Gaussian kernel. The latter areas are defined in Fig. 2 (labeled as "filament B" and "filament C" in Fig. 5 of Durret et al. 2016). We note that the filament detected from WL is included in "filament B", and that "filament C", lying to the south of the previous structure, is only weakly detected with lensing compared to the other structures (the $3 \sigma$ detections in Martinet et al. 2016).

The RS GLFs for the six different areas are presented in Fig. 8, in the F814W and F606W bands, and the parameters of the Schechter fits are summarized in Table 2. The different subareas are populated with 1127, 735, and $1035 \mathrm{RS}$ galaxies for "Cluster $1 \mathrm{Mpc}$ ", "Cluster $807 \mathrm{kpc}$ ", and "Cluster WL" respectively, and with 254, 1640, and 1423 galaxies within the RS color cut for "Filament WL", "Filament B", and "Filament C", respectively. The GLFs are however normalized to $1 \mathrm{deg}^{2}$. Since we have only two optical bands, we assume that the RS cut is a good selection of galaxies lying at the cluster redshift. We relax this assumption later in this section.

We first compare the results of the RS GLF computed in the $807 \mathrm{kpc}$ radius with those of Martinet et al. (2017) for the same cluster. We find a perfect agreement between the two studies in both bands. Quantitatively, we find faint-end slopes $\alpha=-0.80 \pm$ 0.07 and $\alpha=-1.08 \pm 0.09$ in F814W and F606W, respectively, while Martinet et al. (2017) found $\alpha=-0.84 \pm 0.44$ and $\alpha=$ $-1.11 \pm 0.51$. Although the values are almost identical, we note the tremendous gain in precision, explained by the extension of the fit to galaxies almost four magnitudes fainter.

The GLF in the cluster WL contours is almost identical to that of the inner part of the cluster, showing that more complex extended contours defined by WL probe the same galaxy population as that of the cluster core. In the WL contours of the filamentary structure, we note a drop in the galaxy density but the shape of the GLF remains the same. This last result is a hint that the structure selected through its lensing effect is probably a pre-processed group embedded in the filament, which already contains a RS and is falling onto the cluster. Although we do not know what a filament GLF looks like, we expect it to lie somewhere between the field and cluster GLFs. We also show the GLFs of field galaxies with the same color selection as the RS, 

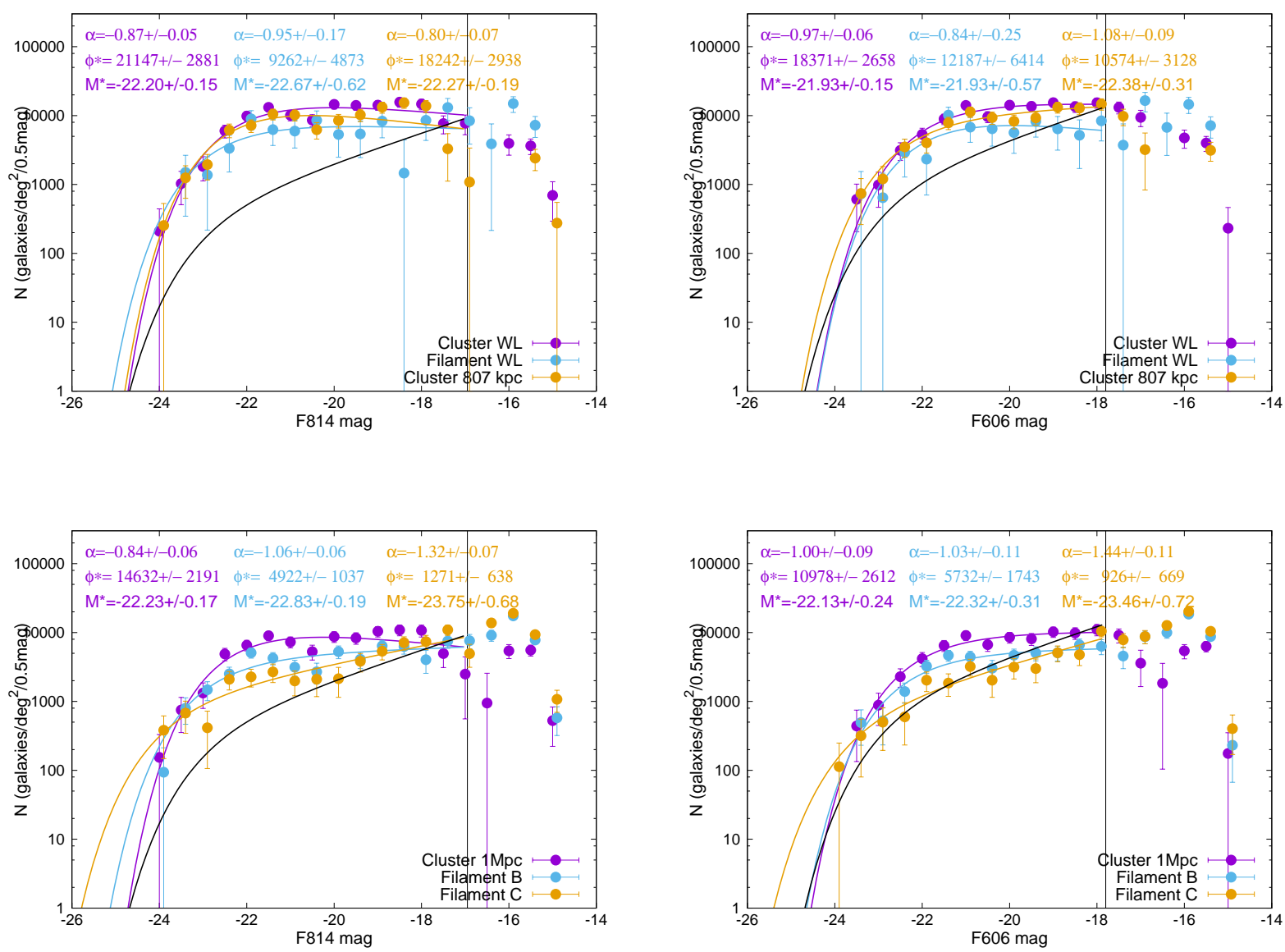

Fig. 8. Red sequence GLFs in the F814W (left) and F606W (right) filters. Top: GLFs centered on the cluster in a $807 \mathrm{kpc}$ radius (yellow), on the cluster WL contours (violet), and on the southeastern filament WL contours (cyan). Bottom: GLFs centered on the cluster in a $1 \mathrm{Mpc}$ radius (violet), and in the optically detected filaments B (cyan) and C (yellow). The different curves are the Schechter fit to the data, and the parameters of the fit are displayed in the same color. In both plots the black curve corresponds to the GLF that we would observe if we were selecting field galaxies and incorrectly assuming that they lie at the cluster redshift. The black vertical line corresponds to the completeness magnitude limit.

Table 2. Schechter fit parameters for RS GLFs for the six different areas.

\begin{tabular}{|c|c|c|c|c|c|c|}
\hline & \multicolumn{3}{|c|}{$\mathrm{F} 814 \mathrm{~W}$} & \multicolumn{3}{|c|}{ F606W } \\
\hline & $\begin{array}{c}\alpha \\
\text { (dimensionless) }\end{array}$ & $\begin{array}{c}M^{*} \\
\text { (mag) }\end{array}$ & $\begin{array}{c}\phi^{*} \\
\left(\text { galaxies deg }{ }^{-2} \text { ) }\right.\end{array}$ & $\begin{array}{c}\alpha \\
\text { (dimensionless) }\end{array}$ & $\begin{array}{c}M^{*} \\
\text { (mag) }\end{array}$ & $\begin{array}{c}\phi^{*} \\
\left(\text { galaxies } \mathrm{deg}^{-2} \text { ) }\right.\end{array}$ \\
\hline Cluster 807 kpc & $-0.80 \pm 0.07$ & $-22.27 \pm 0.19$ & $18242 \pm 2938$ & $-1.08 \pm 0.09$ & $-22.38 \pm 0.31$ & $10574 \pm 3128$ \\
\hline Cluster $1000 \mathrm{kpc}$ & $-0.84 \pm 0.06$ & $-22.23 \pm 0.17$ & $14632 \pm 2191$ & $-1.00 \pm 0.09$ & $-22.13 \pm 0.24$ & $10978 \pm 2612$ \\
\hline Cluster WL & $-0.87 \pm 0.05$ & $-22.20 \pm 0.15$ & $21147 \pm 2881$ & $-0.97 \pm 0.06$ & $-21.93 \pm 0.15$ & $18371 \pm 2658$ \\
\hline Filament WL & $-0.95 \pm 0.17$ & $-22.67 \pm 0.67$ & $9262 \pm 4873$ & $-0.84 \pm 0.25$ & $-21.93 \pm 0.57$ & $12187 \pm 6414$ \\
\hline Filament B & $-1.06 \pm 0.06$ & $-22.83 \pm 0.19$ & $4922 \pm 1037$ & $-1.03 \pm 0.11$ & $-22.32 \pm 0.31$ & $5732 \pm 1742$ \\
\hline Filament C & $-1.32 \pm 0.07$ & $-23.75 \pm 0.68$ & $1271 \pm 638$ & $-1.44 \pm 0.11$ & $-23.46 \pm 0.72$ & $926 \pm 669$ \\
\hline
\end{tabular}

Notes. $\alpha, M^{*}$, and $\phi^{*}$ correspond to the faint end slope, the characteristic magnitude, and the normalization, respectively.

and assuming they lie at the cluster redshift. This last assumption is false but allows us to see what the RS GLF would look like if we misinterpret a field region for a filament. We see that it would give rise to a lower bright galaxy density and to a steeper faint end than what is observed in this potential group of galaxies. The results in the F606W filter for the WL-defined structures are very similar to those in the F814W filter, with a slightly lower number of bright galaxies above the field.

We now look at the GLFs defined within the optical contours of Durret et al. (2016). The cluster in a $1 \mathrm{Mpc}$ radius presents a typical GLF for that redshift, in good agreement with those computed both in a $807 \mathrm{kpc}$ radius, and within the WL contours. The
GLF in filament B shows a lower density than that within the WL contours, but also a slight steepening of the faint end, intermediate between the cluster and field behaviors in the F814W filter. This comforts us in our interpretation of the extended structure detected in the optical being a filament with a WL-detected overdensity corresponding to a group within the filament. Filament $\mathrm{C}$ has an even steeper faint end, very close to what we would get if it was made of field galaxies, but with a significant overdensity at the bright end in F814W. The results are similar in F606W, although the bright overdensity is less pronounced for filament $\mathrm{B}$, and filament $\mathrm{C}$ shows no difference from the case where we select red field galaxies. It is therefore possible that 

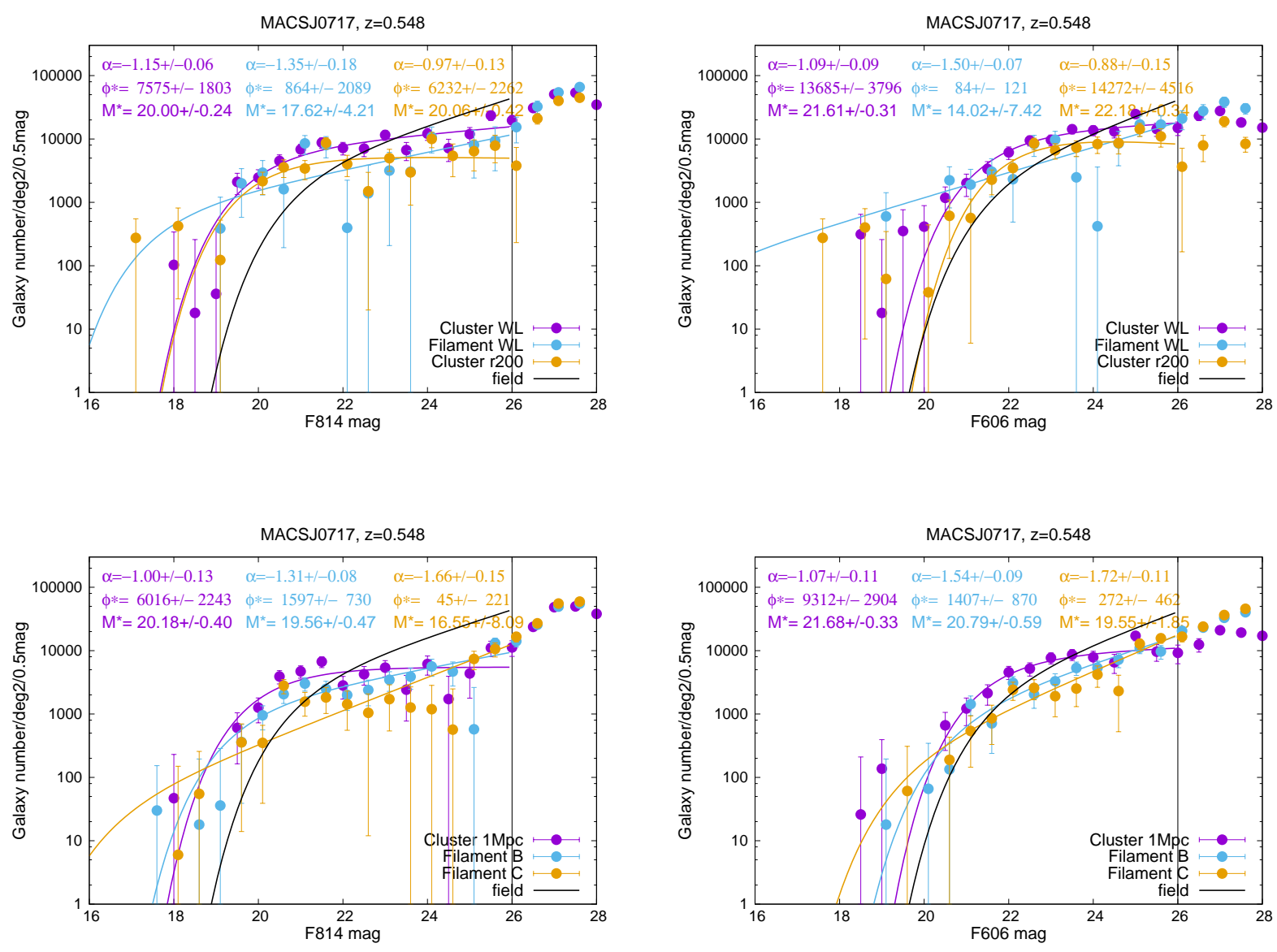

Fig. 9. As in Fig. 8 but for all galaxies (i.e., not only RS galaxies) in the F814W (left) and F606 (right) apparent magnitudes.

filament $\mathrm{C}$ does not correspond to a filament but more likely simply to a few red bright galaxies superimposed on the field, a hypothesis which is also suggested by its low WL detection. The differences between the F606W and F814W bands can be understood considering the restframe wavelength that is probed. At a redshift of $z=0.5458$, the F606W filter corresponds to the restframe blue band, and the absence of bright galaxies in this filter highlights a low star-forming efficiency in the filamentary structures. In F814W however, we observe rest-frame wavelengths corresponding to the $g$ and $r$ filters, and see passive galaxies that populate the cluster RS.

Since we know that the RS may not be a good way of selecting filament galaxies, for example because they could correspond to field galaxies which should not lie at the cluster redshift, we also compute GLFs for all galaxies in apparent magnitudes, in F814W and F606W. The results (see Fig. 9) are very similar to what we find with the RS GLFs but with larger error bars due to a larger dilution by field galaxies. The disappearance of bright galaxies from F814W to F606W remains for filament $\mathrm{B}$, and filament $\mathrm{C}$ shows no significant overdensity of bright galaxies in any band compared to the field.

What comes out of this study is that filament B shows a GLF between that of a cluster and that of the field, with an embedded pre-processed group detected in WL, and that filament $\mathrm{C}$ is a much poorer structure that might instead correspond to field galaxies. Finally, the difference between the F814W and F606W filters (especially in the case with all galaxies) tends to show that the galaxies in filaments close to clusters are preferentially passive rather than star forming.

\subsection{Selecting blue galaxies at the cluster redshift}

Without spectroscopic or photometric redshift information for all the galaxies, selecting objects inside a cosmological filament is a difficult task. In our case, having two magnitude bands allows for example to select red galaxies if we assume that galaxy populations in filaments are already preprocessed and show a red sequence. This assumption is probably true within the cluster-infalling groups embedded in the filaments, but is more questionable for early-type galaxies that are not group members, a population which is probably not dominant in the filaments.

If we now consider late-type galaxies, trying to select them in a filament by using the cluster blue cloud characteristics is probably impossible and unjustified. In an attempt to apply another way to select such blue galaxies in the filament detected in the MACS J0717 FoV, we chose to estimate the spatial distribution of these galaxies. Our present assumption is that their spatial distribution is not too different from that of red galaxies. Filaments of galaxies are structures of low mass and concentration, without a very dense intracluster medium (X-ray emission of filaments is weak), and their potential well should therefore not differentially affect their red and blue galaxies as a function of their mass. This hypothesis is also supported by the studies of the two-point correlation function of field galaxies. At similar redshifts, de la Torre et al. (2011; see their Fig. 12) show that blue and red galaxies have very similar two-point correlation signals for correlation lengths lower than $0.2 \mathrm{Mpc}$ and larger than $0.8 \mathrm{Mpc}$ (to be compared with the typical size of the filaments studied here: $\sim 2 \times 4 \mathrm{Mpc}$ ). 

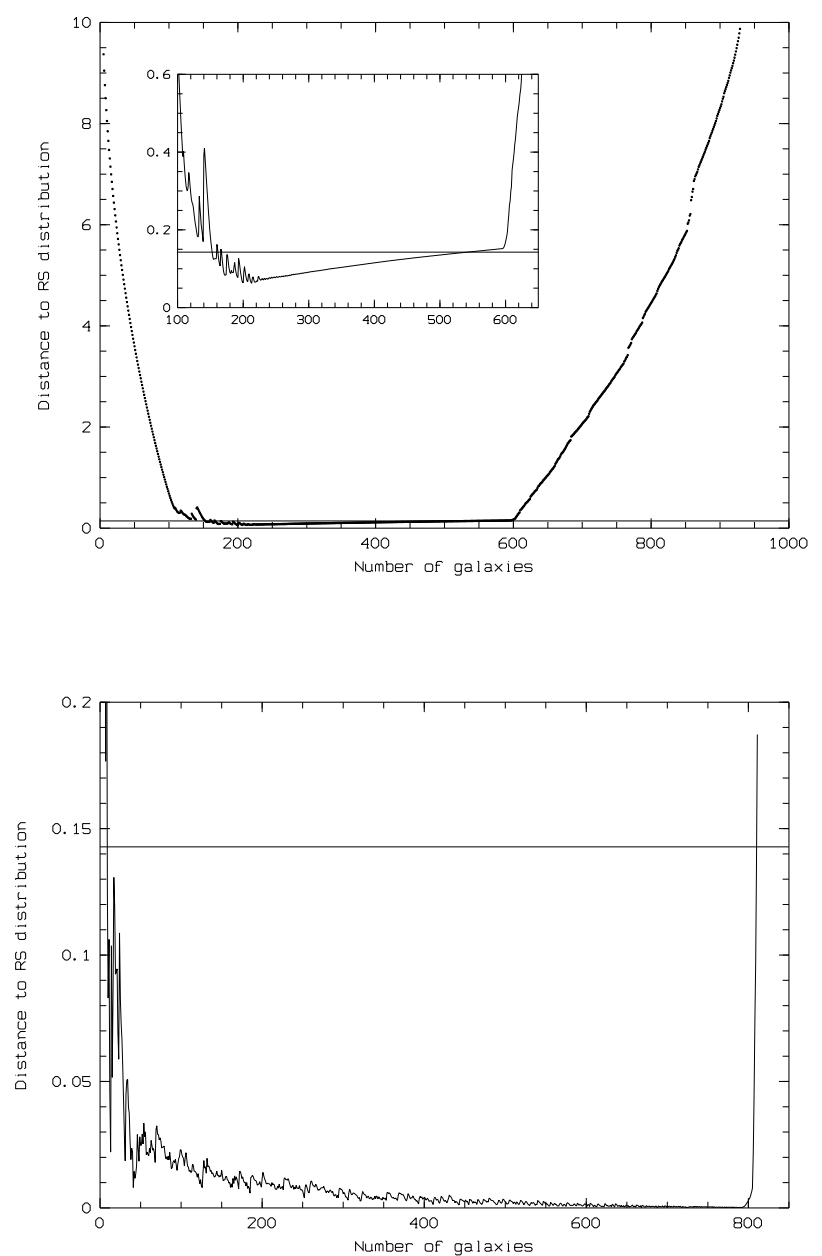

Fig. 10. Distance D between RS and ORS galaxy samples vs. number of galaxies in the ORS galaxy sample. The horizontal line is the typical uncertainty in the D measurement from Adami \& Mazure (1999). Top: filament B. Bottom: filament C, with the inner plot showing a zoom on the region of interest.

We therefore chose to use the Minimal Spanning Tree (MST) technique. This tool allows spatial distributions of points to be characterized by tracing the tree of minimal length linking all the considered points (Adami \& Mazure 1999). For a given set of points, the tree of minimal length is not unique, but the histogram of the lengths of its branches is unique. This histogram therefore fully characterizes a given distribution of points.

In our case, we considered galaxy distributions on the sky (a 2D approach) because we have no redshift information. As shown in Adami \& Mazure (1999), the optimal set of statistic descriptors of the histograms of branch lengths is the mean, $\mu$, the rms, $\sigma$, and the skewness, $\mathcal{S}$, of the branch lengths.

The general goal of our attempt is to find the nonred sequence galaxy populations with a $2 \mathrm{D}$ spatial distribution as close as possible to that of the RS galaxy population inside the area of the filament candidates. To do this we use the following procedure.

1. We select all galaxies present on the line of sight of filaments B and C (see Fig. 2).

2. We select galaxies in the RS for these two areas, and compute $\mu, \sigma$, and $\mathcal{S}$ for the branch lengths of their MSTs. These RS galaxies are therefore supposed to be filament members.

3. We select in the same areas all galaxies outside of the red sequence (ORS). These samples include blue filament mem- ber galaxies plus foreground and background galaxies. If our assumption is true, the MST built on the blue filament member galaxies should have the same $\mu, \sigma$, and $\mathcal{S}$ as the MST of RS galaxies.

4. The goal is then to find within each filament candidate the ORS galaxy sub-population that is the closest to the RS galaxy population. This is technically done by computing a quadratic distance between the two populations in parameter space (Adami \& Mazure 1999):

$D=\sqrt{\left(\mu_{\mathrm{RS}}-\mu_{\mathrm{ORS}}\right)^{2}+\left(\sigma_{\mathrm{RS}}-\sigma_{\mathrm{ORS}}\right)^{2}+\left(\mathcal{S}_{\mathrm{RS}}-\mathcal{S}_{\mathrm{ORS}}\right)^{2}}$,

5. We start with the total ORS galaxy sample and we search for a single galaxy to remove from the sample in order to have the largest diminution of $D$. This galaxy is supposed to be a foreground or background object, and is removed from the running ORS galaxy sample.

6. Step 5 is repeated iteratively. This allows us to draw Fig. 10, where we show the value of $D$ as a function of the number of removed galaxies. As expected, the curves show a minimum value of $D$, corresponding to the ORS galaxy population that is the most similar to the RS galaxy population in terms of spatial distribution. However, there are some uncertainties in our calculations, due to the intrinsic statistical errors in the estimations of $\mu, \sigma$, and $\mathcal{S}$. This results in a typical error bar shown as the horizontal line in Fig. 10 (see Adami \& Mazure 1999 for the estimate).

7. We can then finally use Fig. 10 to define three sub-samples within the ORS galaxy populations.

- The maximal sample (MAX hereafter): largest possible ORS galaxy sample with a $D$ value lower than the typical uncertainty.

- The minimal sample (MIN hereafter): smallest possible ORS galaxy sample with a $D$ value lower than the typical uncertainty.

- The optimal sample (OPT hereafter): ORS galaxy sample with the lowest possible $D$ value.

\subsection{Galaxy luminosity functions of blue galaxies}

Figure 10 clearly shows that for filament C, the ORS galaxy sample is very similar in terms of spatial distribution to the RS galaxy sample, whatever the selection within the ORS sample (D is nearly always lower than the typical uncertainty). This could mean that defining a red sequence in filament $C$ is meaningless.

Filament B shows a clearer tendency of the ORS sample to be similar to the RS sample only between 150 and 600 galaxies. As compared to the initial $\sim 930$ galaxies along the line of sight within the ORS sample, this means that we need to remove at least $\sim 35 \%$ of the galaxies along the line of sight in order to obtain similar spatial distributions between ORS and RS samples.

We analyzed the filament of galaxies detected between the A222 and A223 galaxy clusters in the same way (Durret et al. 2010). Despite being only poorly sampled with spectroscopy (only five galaxy spectroscopic redshifts in the ORS sample: four within the filament and one outside), we find that the galaxies outside of the filament are the first to be removed in the process (well before $\mathrm{D}$ reaches its minimal value) while the galaxies inside the filament start to be removed just before $D$ reaches its minimal value.

We therefore decided to that the ORS MAX sample is the galaxy sample representing the filament blue galaxies in the best 

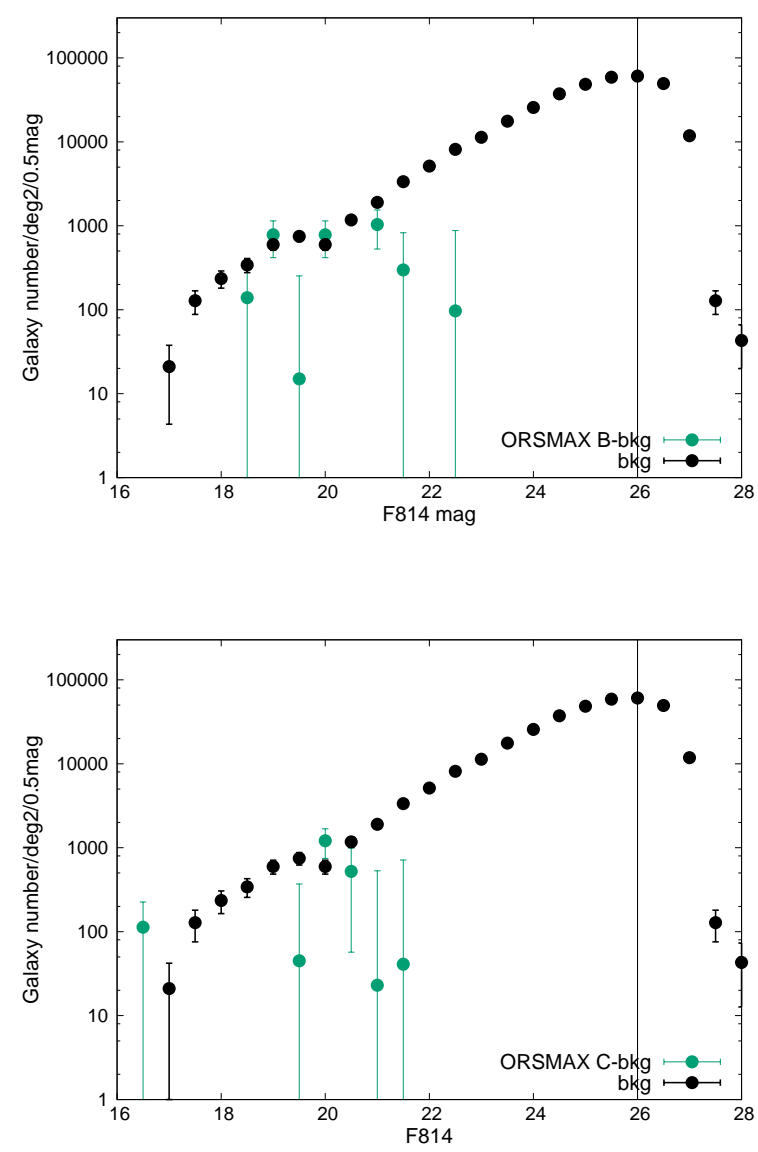

Fig. 11. F814W apparent magnitude luminosity functions of samples of blue galaxies in filaments B (top) and C (bottom). Black disks represent the field, and green disks the filament blue samples after field subtraction.

possible way. With this sample, we compute the resulting galaxy luminosity function following the method described in Sect. 4.1 (see Fig. 11). Since we do not consider RS galaxies, it is not possible to compute an accurate $k$-correction in this case. In addition this approach requires that background galaxies be treated differently. We apply a color cut to select field galaxies that lie outside of the cluster RS, and we also re-weight field galaxy counts by the ratio of the number of galaxies in the ORS catalog to the number of galaxies in the ORS MAX catalog to account for the dilution in the blue galaxy selection process.

We derived the GLFs of these blue galaxies in the F606W and $\mathrm{F} 814 \mathrm{~W}$ bands for filaments $\mathrm{B}$ and $\mathrm{C}$. The results are relatively noisy, but one interesting feature is an excess of bright blue galaxies in filament $\mathrm{B}$, with about twice as many blue galaxies brighter than $M^{*}$ than in the field. This suggests that a large group or small cluster, rich in bright blue galaxies, resides in region B and is merging with the main cluster, MACS J0717. This group would be demarcated by the cyan contour in Fig. 2.

\section{Galaxy alignments}

To gain more insight into the filament embedding MACS J0717.5+3745, this section aims at assessing whether galaxies located in this structure show any preferential orientation of their major axis or instead have random orientations. As introduced in Sect. 1 , this is motivated by previous findings of preferential directions for the orientations of filament galaxies - especially for some galaxy types or classes - relative to the orientation of the filament ridge, be it in observations at low- $z$ (e.g., Tempel \& Libeskind
2013; Tempel et al. 2013; Zhang et al. 2015; Chen et al.2019, and references therein) or in N-body and hydrodynamic simulations (e.g., Chen et al. 2015; Codis et al. 2015; Ganeshaiah Veena et al. 2018, and references therein). In both types of work, the underlying physical motivation for such alignments lies in tidal torque theory and mergers, as amply discussed in all the latter-mentioned references. The assessment of this behavior is expected to put some constraints on galaxy and structure formation theories, and alignments have also been used to develop alternative methods of detecting filaments in the cosmic web around clusters (Rong et al. 2016). However, up to now, disparate results have been found, and the picture is far from clear. For instance, in contrast with previous results for the local Universe based on SDSS data, the recent work by Krolewski et al. (2019) uses MaNGA kinematic maps and finds no evidence for alignment between galaxy spins and filament directions. Our study on the filaments connected with MACS J0717.5+3745 intends to add up to the observational determinations of galaxy orientations in filaments, this time in a structure at $z \sim 0.5$.

Since filament B seems to be dominated by a group of galaxies (possibly located at the infall region of the cluster), as already discussed in the previous sections, we restrict our analysis to galaxy orientations in filament $\mathrm{C}$. We therefore selected the frames from the HST mosaic covering this region only (the green ellipse in Fig. 2). We note that filament $\mathrm{C}$ is almost totally covered by these observations that leave out only a small percentage (7.9\%) of its southern edge.

Because astrometry is accurate and homogeneous in this region, we can now adopt a slightly different approach from Sect. 4: as before, detections were made individually on each image of the HST mosaic, but we now run SExtractor in double mode so as to directly obtain colors for the galaxies. We first ran this software on the deeper, less noisy F814W images to detect sources, and compute their magnitude and peak surface brightness. Detection, background, deblending, and aperture parameters were optimized especially for all objects that clearly stand out from the background, without aiming to achieve a thorough detection of faint galaxies that could be confused with noise or lost in brighter background areas. In particular, we studied the apertures for photometry in the images by eye to make sure that their extent was sufficient to fully cover galaxies down to the sky level and that their orientation appeared correct. The hand-made masks covering all problematic areas (such as saturated stars, their bright spikes, and image edges) were used to discard spurious and contaminated detections at this stage and in the remainder of the analysis.

For each image, we next selected stars in a magnitudemaximum surface brightness plane (MU_MAX versus $\mathrm{MAG}_{-}$ AUTO), and used the catalog of these objects as input for a second run of SExtractor to obtain the necessary files for PSFEx (Bertin 2011), previously verifying that these stars were well spread all over the image. The PSF model computed with PSFEx for each image was saved, and fed into SExtractor in a third run for all images, now carried out in the ASSOC mode, choosing a de Vaucouleurs plus a disk model to fit all sources. This allowed us to compute PSF-corrected magnitudes (MAG_AUTO, which include the correction for Galactic extinction according to Schlafly \& Finkbeiner 2011), position angles, and ellipticities for all objects in all images in the F814W filter. Finally, to compute galaxy colors, we performed a final run of SExtractor in double mode, using F814W as the detection images while magnitudes were now measured in the corresponding F606W images (adapting all necessary parameters such as magnitude zero point, extinction correction, and gain). 


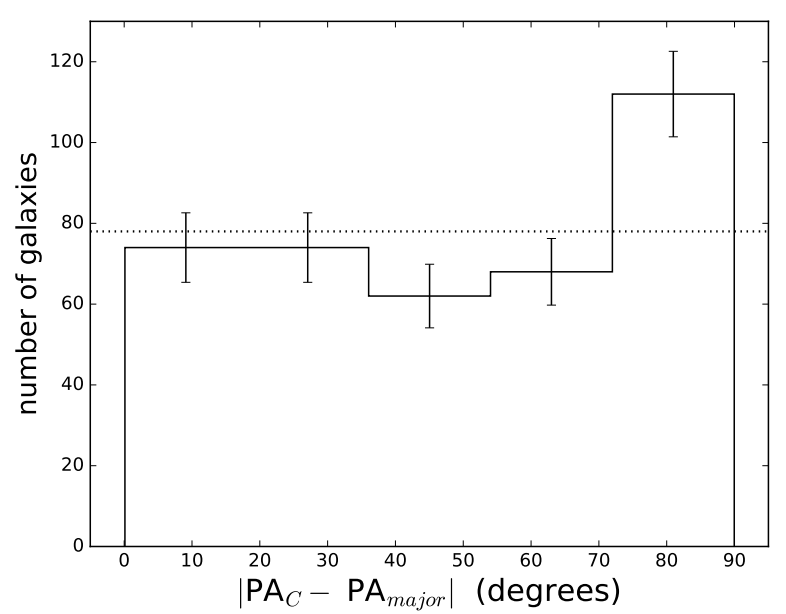

Fig. 12. Distribution of the angles (in absolute value) subtended by the orientation of filament $\mathrm{C}$ and the orientation of the position angles of the $390 \mathrm{RS}$ galaxies located in the same filament (within the $3 \sigma$ density contours - see text). Error bars assume Poissonian statistics and the dotted line represents the average number of galaxies per bin.

Catalogs obtained for all frames in the two filters were concatenated, and double entries were excluded, based on the criteria described in the previous section, for objects located where adjacent frames overlap. Finally, stars were purged from the catalog (based on their position in the MAG_AUTO - MU_MAX plane), which was further limited to include only objects brighter than $25 \mathrm{mag}$ in F814W. This last point minimizes the number of objects with large errors in the determination of the position angle. At this stage, we selected galaxies with positions (RA, DEC) within the green ellipse of Fig. 2 (i.e., the $3 \sigma$ density contours that delineate filament $\mathrm{C}$ as defined in Durret et al. 2016).

The next step would be to identify all galaxies within this filament along the line-of-sight. As before, and in the absence of abundant spectroscopic coverage or photometric redshifts for this area, we used the cluster red sequence to select galaxies in the filament, assuming through this color indication that they lie at the same distance as cluster galaxies. This produced a sample of 390 galaxies. Among these, there remained a percentage of field galaxies (up to $\sim 59 \%$, as estimated from the field counts; Sect. 4), but since we did not expect these objects to have a particular orientation, their presence was not expected to affect the results.

Figure 12 shows the absolute value of the angle between the direction of filament $\mathrm{C}$ (which is oriented north-south) and the position angle of the major axis for all 390 galaxies included in the previously defined sample. There seems to be an excess of red sequence galaxies with their major axis oriented perpendicularly to the direction of the filament, although this is a low-significance trend (a departure of less than $3 \sigma$ from the mean behavior). The Anderson-Darling test issues a significance level of 4\% when comparing the two distributions of Fig. 12. According to the classical interpretation of the $p$-value in such statistical tests, a significance level of $4 \%$ is usually considered to be sufficiently low to conclude that the null hypothesis (i.e., the two distributions are alike) is clearly disfavored. However, this value lies at the borderline (usually taken to be $5 \%$ ) between accepting or rejecting the null hypothesis; if we were to take the error bars into account, then the expected significance level would likely increase, rendering the test result inconclusive.

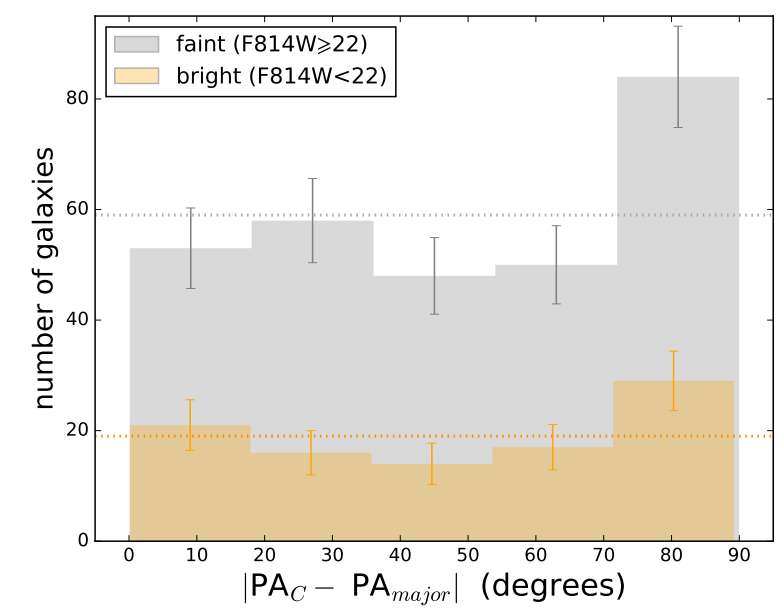

Fig. 13. Same as Fig. 12 but splitting the sample according to apparent magnitude in the F814W band: there are 97 bright galaxies and 293 faint galaxies.

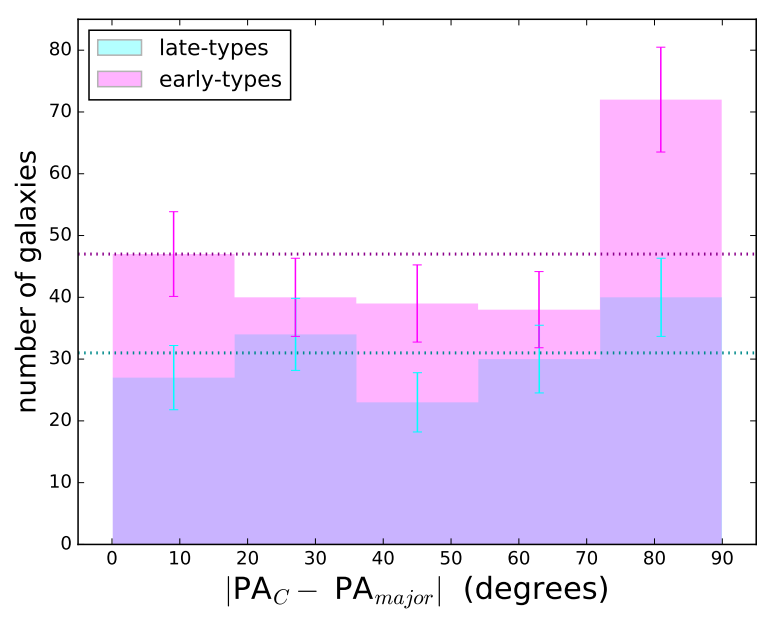

Fig. 14. Same as Fig. 12 but splitting the sample by galaxy type: 236 early-type galaxies against 154 late-type galaxies.

To understand however whether the trend seen in Fig. 12 is caused by a specific class of RS galaxies, we split the sample by type and brightness. As a proxy for the morphological type, we considered the bulge-to-total flux ratio in the F814W band, setting the frontier at 0.35 for this quantity as in Simard et al. (2009). As for brightness, we set the separation at 22 mag in F814W, so as to keep a reasonable number of objects in the bright set (different alternative limits were tested without relevant changes to the outcome). For this new brightness cut, the percentage of field galaxies possibly contaminating the sample decreases to about $46 \%$. Figures 13 and 14 show the results after separating the sample according to each of these two criteria.

Figure 13 shows that it is faint galaxies (which are also dominant in number) that are preferentially oriented perpendicularly to the filament, and these are likely of early-type (Fig. 14). We find no clear indication for a preferential alignment for either bright or late-type galaxies that are much less numerous. However, we note that a robust analysis based on morphologies is hampered both by our crude classification and by the known difficulty in determining position angles for pure ellipticals (which likely makes up a certain percentage of our early-type galaxies) using photometric data only. 
It is difficult to compare our result with other observational determinations, and also with the published results based on simulations, due to our selection: restricting our analysis to RS galaxies limits our capacity to infer definite conclusions. Still, our study of region $\mathrm{C}$ provides a low-significance indication of an excess of faint galaxies that have their major axis aligned along a direction perpendicular to the filament.

To test the robustness of this result, we repeated the same analysis by restricting our sample to within the $4 \sigma$ density contour region defined by Durret et al. (2016), without any relevant change to the results. We further consider that any errors in the determination of the position angle, likely to affect fainter galaxies more severely, should not be responsible for the appearance of a specific trend. Finally, if we abandon our selection criteria, and use all available spectroscopic data (as in NED) for filament $\mathrm{C}$ instead, we are left with 61 galaxies with a redshift compatible with the cluster redshift range, i.e., [0.530,0.560] (Durret et al. 2016); this is too small a sample for our objectives, further plagued with incompleteness in terms of limiting magnitude for the spectroscopic coverage. Results are thus noisier and relatively inconclusive.

In summary, our results for region $\mathrm{C}$ point to there being no preferential orientation of bright RS galaxies, whereas a preferential orientation of faint RS galaxies might exist, in which the major axes of galaxies lie mainly perpendicular to the direction of the filament. Such a result is of low significance however (below $3 \sigma$ from random alignment). Nonetheless, if we consider this result, it seems to be in contrast with what authors have been finding at lower redshifts based on SDSS data, where small but significant alignment in the direction parallel to the orientation of nearby filaments was found (Tempel \& Libeskind 2013; Tempel et al. 2013; Zhang et al. 2015; Chen et al. 2019, and other works of these teams).

Three reasons could be proposed to explain this discrepancy: (i) there is no filament in region $C$ (as hinted at by the results of Sect. 4); (ii) the filament does exist (as inferred by Durret et al. 2016, even if it is not a very dense structure given its low-significance detection in weak lensing and absence of X-ray emission), but we are unable to correctly identify its member galaxies; or (iii) the filament does exist, and we are correctly sampling its population (even if only a subset of it), and the fact that we do not obtain the trends in orientation that are found in low-z systems remains to be understood. This could be a real result, explained by galaxy evolution within the filaments or simply by the intrinsic variety of filament properties, or be something induced by errors, either in our assessment of the position angles or in the estimates provided by other authors, possibly worsened by the lower-quality SDSS images. Interestingly enough, in a search for possible radial alignments in massive clusters located at $z>0.5$, and observed with HST (MACS J0717.5+3745 included), Hung \& Ebeling (2012) found no such alignments, in stark contrast to what is reported for nearby clusters at $z \sim 0.1$ using SDSS data. These authors discuss possible explanations for the discrepancy, invoking either evolutionary effects or the "presence of systematic biases in the analysis of SDSS imaging data that cause at least partly spurious alignment signals". It is thus plausible that similar causes could explain the lack of agreement between our HST-based results for a $z \sim 0.5$ filament and trends obtained for lower-redshift filaments in SDSS data. Finally, future determinations of galaxy orientations in this filament, carried out with the aid of spectroscopic data, to select only galaxies at the cluster redshift and in other filaments around $z=0.5$, will be of great help in disentangling this issue.

\section{Conclusions}

We analyzed the properties of the cosmic filament of MACS J0717 by computing the GLF in different regions (B and C). While the RS GLF of region $C$ is that of a poor structure close to that of field galaxies, the RS GLF of region $B$ is much richer and typical of a galaxy group. This is consistent with the presence of WL contours in area B corresponding to a denser area. Recent work has also detected this structure and estimated its mass within $150 \mathrm{kpc}\left(M_{150}=(2.28 \pm 0.24) \times 10^{13} M_{\odot}\right.$, Jauzac et al. 2018). When looking at the GLFs of blue galaxies, an excess of bright blue galaxies in region B leads to the same conclusion: a rich galaxy group moving along the cosmic filament to merge with MACS J0717. Merging phenomena in the filament could therefore take place preferentially for massive blue galaxies, with some gas remaining, allowing them to remain blue.

We also studied the orientation of RS galaxies in filament $\mathrm{C}$, finding no preferential orientation of bright RS galaxies. A preferential orientation perpendicular to the direction of the filament for faint galaxies might exist but is below the $3 \sigma$ level of significance from a random distribution, making it difficult to draw conclusions.

We used our new software DAWIS to detect and estimate the ICL of the core of MACS J0717 in the HFF, finding ICL fractions coherent with the literature. The fact that we detect almost no ICL in the UV rest frame of MACS J0717 indicates that there must be little star formation in the ICL. This is also confirmed by the fact that the ICL in the F105W band (that contains the $\mathrm{H} \alpha$ line) is also rather weak. This result agrees with results based on integral field spectroscopy with VLT/MUSE by Adami et al. (2016) for a cluster (XLSSC 116) at a similar redshift (see their Table 2).

We tried to detect large diffuse sources in the filament that could be associated with IFL, without success. We found a single system of strongly disturbed galaxies with obvious tidal streams lying inside the WL contours of the galaxy group falling into MACS J0717. This supports the ICL formation scenario in which such galaxy groups form IGL through tidal stripping and mergers, and then fall into larger structures, mixing their IGL into the ICL of the larger structure.

Acknowledgements. We acknowledge long-term support from CNES. C. Lobo thanks the IAP for inviting her for a week and further acknowledges support by Fundação para a Ciência e a Tecnologia (FCT) through national funds (UID/FIS/04434/2013) and by FEDER - Fundo Europeu de Desenvolvimento Regional through COMPETE2020 - Programa Operacional Competitividade e Internacionalização (POCI-01-0145-FEDER-007672), and by FCT/MCTES through national funds (PIDDAC) via grant UID/FIS/04434/2019. N. Martinet acknowledges support from a CNES fellowship. M. Jauzac acknowledges support by the Science and Technology Facilities Council (grant number ST/L00075X/1). This research has made use of the NASA/IPAC Extragalactic Database (NED), which is operated by the Jet Propulsion Laboratory, California Institute of Technology, under contract with the National Aeronautics and Space Administration. We acknowledge Mark Taylor for the development of TOPCAT (Taylor 2005).

\section{References}

Adami, C., \& Mazure, A. 1999, A\&AS, 134, 393

Adami, C., Slezak, E., Durret, F., et al. 2005, A\&A, 429, 39

Adami, C., Durret, F., Guennou, L., \& Da Rocha, C. 2013, A\&A, 551, A20

Adami, C., Pompei, E., Sadibekova, T., et al. 2016, A\&A, 592, A7

Aguerri, J. A. L., Castro-Rodríguez, N., Napolitano, N., Arnaboldi, M., \&

Gerhard, O. 2006, A\&A, 457, 771

Andreon, S. 2006, MNRAS, 369, 969

Arnouts, S., Cristiani, S., Moscardini, L., et al. 1999, MNRAS, 310, 540 
Bertin, E. 2011, in Astronomical Data Analysis Software and Systems XX, eds I. N. Evans, A. Accomazzi, D. J. Mink, \& A. H. Rots, ASP Conf. Ser., 442 435

Bertin, E. 2013, Astrophysics Source Code Library [record ascl:1301.001]

Bertin, E., \& Arnouts, S. 1996, A\&AS, 117, 393

Bijaoui, A., \& Rué, F. 1995, Signal Process., 46, 345

Bond, J. R., Kofman, L., \& Pogosyan, D. 1996, Nature, 380, 603

Brammer, G. B., van Dokkum, P. G., Franx, M., et al. 2012, ApJS, 200, 13

Burke, C., Collins, C. A., Stott, J. P., \& Hilton, M. 2012, MNRAS, 425, 2058

Byrd, G., \& Valtonen, M. 1990, ApJ, 350, 89

Capaccioli, M., \& de Vaucouleurs, G. 1983, ApJS, 52, 465

Chen, Y.-C., Ho, S., Tenneti, A., et al. 2015, MNRAS, 454, 3341

Chen, Y.-C., Ho, S., Mandelbaum, R., et al. 2017, MNRAS, 466, 1880

Chen, Y.-C., Ho, S., Blazek, J., et al. 2019, MNRAS, 485, 2492

Codis, S., Gavazzi, R., Dubois, Y., et al. 2015, MNRAS, 448, 3391

Contini, E., De Lucia, G., Villalobos, Á., \& Borgani, S. 2014, MNRAS, 437, 3787

Contini, E., Yi, S. K., \& Kang, X. 2018, MNRAS, 479, 932

Da Rocha, C., \& Mendes de Oliveira, C. 2005, MNRAS, 364, 1069

Da Rocha, C., Ziegler, B. L., \& Mendes de Oliveira, C. 2008, MNRAS, 388, 1433

de Jong, R. S. 2008, MNRAS, 388, 1521

de la Torre, S., Meneux, B., De Lucia, G., et al. 2011, A\&A, 525, A125

De Lucia, G., Poggianti, B. M., Aragón-Salamanca, A., et al. 2004, ApJ, 610, L77

De Lucia, G., Poggianti, B. M., Aragón-Salamanca, A., et al. 2007, MNRAS, 374, 809

De Propris, R., Phillipps, S., \& Bremer, M. N. 2013, MNRAS, 434, 3469

DeMaio, T., Gonzalez, A. H., Zabludoff, A., et al. 2018, MNRAS, 474, 3009

Diego, J. M., Broadhurst, T., Zitrin, A., et al. 2015, MNRAS, 451, 3920

Dietrich, J. P., Werner, N., Clowe, D., et al. 2012, Nature, 487, 202

Dubois, Y., Pichon, C., Welker, C., et al. 2014, MNRAS, 444, 1453

Durret, F., Laganá, T. F., Adami, C., \& Bertin, E. 2010, A\&A, 517, A94

Durret, F., Laganá, T. F., \& Haider, M. 2011, A\&A, 529, A38

Durret, F., Márquez, I., Acebrón, A., et al. 2016, A\&A, 588, A69

Ebeling, H., Barrett, E., \& Donovan, D. 2004, ApJ, 609, L49

Eckert, D., Jauzac, M., Shan, H., et al. 2015, Nature, 528, 105

Fukugita, M., Shimasaku, K., \& Ichikawa, T. 1995, PASP, 107, 945

Ganeshaiah Veena, P., Cautun, M., van de Weygaert, R., et al. 2018, MNRAS, 481,414

Gonzalez, A. H., Zabludoff, A. I., \& Zaritsky, D. 2005, ApJ, 618, 195

Gregg, M. D., \& West, M. J. 1998, Nature, 396, 549

Gu, M., Conroy, C., Law, D., et al. 2018, ApJ, 859, 37

Guennou, L., Adami, C., Da Rocha, C., et al. 2012, A\&A, 537, A64

Hung, C.-L., \& Ebeling, H. 2012, MNRAS, 421, 3229

Ilbert, O., Arnouts, S., McCracken, H. J., et al. 2006, A\&A, 457, 841

Janowiecki, S., Mihos, J. C., Harding, P., et al. 2010, ApJ, 715, 972

Jauzac, M., Jullo, E., Kneib, J.-P., et al. 2012, MNRAS, 426, 3369

Jauzac, M., Eckert, D., Schaller, M., et al. 2018, MNRAS, 481, 2901

Jiménez-Teja, Y., \& Dupke, R. 2016, ApJ, 820, 49

Jiménez-Teja, Y., Dupke, R., Benítez, N., et al. 2018, ApJ, 857, 79

Jiménez-Teja, Y., Dupke, R. A., Lopes de Oliveira, R., et al. 2019, A\&A, 622, A183

Karabal, E., Duc, P.-A., Kuntschner, H., et al. 2017, A\&A, 601, A86

Kartaltepe, J. S., Ebeling, H., Ma, C. J., \& Donovan, D. 2008, MNRAS, 389, 1240

Ko, J., \& Jee, M. J. 2018, ApJ, 862, 95

Krick, J. E., \& Bernstein, R. A. 2007, AJ, 134, 466

Krick, J. E., Bernstein, R. A., \& Pimbblet, K. A. 2006, AJ, 131, 168

Krist, J. E., Hook, R. N., \& Stoehr, F. 2011, in Optical Modeling and Performance Predictions V, Proc. SPIE, 8127, 81270J

Krolewski, A., Ho, S., Chen, Y.-C., et al. 2019, ApJ, 876, 52

Kuutma, T., Tamm, A., \& Tempel, E. 2017, A\&A, 600, L6

Libeskind, N. I., van de Weygaert, R., Cautun, M., et al. 2018, MNRAS, 473, 1195

Limousin, M., Richard, J., Jullo, E., et al. 2016, A\&A, 588, A99
Lotz, J. M., Koekemoer, A., Coe, D., et al. 2017, ApJ, 837, 97

Lucy, L. B. 1974, AJ, 79, 745

Mahajan, S., Raychaudhury, S., \& Pimbblet, K. A. 2012, MNRAS, 427, 1252

Martinet, N., Durret, F., Guennou, L., et al. 2015, A\&A, 575, A116

Martinet, N., Clowe, D., Durret, F., et al. 2016, A\&A, 590, A69

Martinet, N., Durret, F., Adami, C., \& Rudnick, G. 2017, A\&A, 604, A80

Merritt, D. 1984, ApJ, 276, 26

Mihos, J. C. 2004a, in Recycling Intergalactic and Interstellar Matter, eds. P. A. Duc, J. Braine, \& E. Brinks, IAU Symp., 217, 390

Mihos, J. C. 2004b, in Clusters of Galaxies: Probes of Cosmological Structure and Galaxy Evolution, eds. J. S. Mulchaey, A. Dressler, \& A. Oemler, 277

Mihos, J. C., Harding, P., Feldmeier, J., \& Morrison, H. 2005, ApJ, 631, L41

Mihos, J. C., Harding, P., Feldmeier, J. J., et al. 2017, ApJ, 834, 16

Montes, M., \& Trujillo, I. 2018, MNRAS, 474, 917

Montes, M., \& Trujillo, I. 2019, MNRAS, 482, 2838

Moore, B., Katz, N., Lake, G., Dressler, A., \& Oemler, A. 1996, Nature, 379, 613

Moore, B., Lake, G., Quinn, T., \& Stadel, J. 1999, MNRAS, 304, 465

Morishita, T., Abramson, L. E., Treu, T., et al. 2017, ApJ, 846, 139

Murante, G., Arnaboldi, M., Gerhard, O., et al. 2004, ApJ, 607, L83

Murante, G., Giovalli, M., Gerhard, O., et al. 2007, MNRAS, 377, 2

Napolitano, N. R., Pannella, M., Arnaboldi, M., et al. 2003, ApJ, 594, 172

Planck Collaboration VIII. 2013, A\&A, 550, A134

Ricci, M., Benoist, C., Maurogordato, S., et al. 2018, A\&A, 620, A13

Richardson, W. H. 1972, J. Opt. Soc. Am., 62, 55

Rong, Y., Liu, Y., \& Zhang, S.-N. 2016, MNRAS, 455, 2267

Rudick, C. S., Mihos, J. C., \& McBride, C. 2006, ApJ, 648, 936

Rudick, C. S., Mihos, J. C., Frey, L. H., \& McBride, C. K. 2009, ApJ, 699, 1518

Rudick, C. S., Mihos, J. C., \& McBride, C. K. 2011, ApJ, 732, 48

Rudnick, G., von der Linden, A., Pelló, R., et al. 2009, ApJ, 700, 1559

Sandin, C. 2014, A\&A, 567, A97

Sandin, C. 2015, A\&A, 577, A106

Sarron, F., Martinet, N., Durret, F., \& Adami, C. 2018, A\&A, 613, A67

Sarron, F., Adami, C., Durret, F., \& Laigle, C. 2019, A\&A, submitted, [arXiv: 1903.02879 ]

Schechter, P. 1976, ApJ, 203, 297

Schlafly, E. F., \& Finkbeiner, D. P. 2011, ApJ, 737, 103

Shensa, M. J. 1992, IEEE Trans. Signal Process., 40, 2464

Simard, L., Clowe, D., Desai, V., et al. 2009, A\&A, 508, 1141

Skelton, R. E., Whitaker, K. E., Momcheva, I. G., et al. 2014, ApJS, 214, 24

Smail, I., Edge, A. C., Ellis, R. S., \& Blandford, R. D. 1998, MNRAS, 293, 124

Sommer-Larsen, J. 2006, MNRAS, 369, 958

Starck, J.-L., Murtagh, F. D., \& Bijaoui, A. 1998, Image Processing and Data Analysis (New York: Cambridge University Press), 297

Stoica, R. S., Martínez, V. J., Mateu, J., \& Saar, E. 2005, A\&A, 434, 423

Tang, L., Lin, W., Cui, W., et al. 2018, ApJ, 859, 85

Taylor, M. B. 2005, in Astronomical Data Analysis Software and Systems XIV, eds. P. Shopbell, M. Britton, \& R. Ebert, ASP Conf. Ser., 347, 29

Tempel, E., \& Libeskind, N. I. 2013, ApJ, 775, L42

Tempel, E., Stoica, R. S., \& Saar, E. 2013, MNRAS, 428, 1827

Tempel, E., Stoica, R. S., Martínez, V. J., et al. 2014, MNRAS, 438, 3465

Tempel, E., Guo, Q., Kipper, R., \& Libeskind, N. I. 2015, MNRAS, 450, 2727

Tempel, E., Stoica, R. S., Kipper, R., \& Saar, E. 2016, Astron. Comput., 16, 17

Trentham, N., \& Mobasher, B. 1998, MNRAS, 293, 53

Trujillo, I., \& Fliri, J. 2016, ApJ, 823, 123

Vacca, V., Murgia, M., Govoni, F., et al. 2018, MNRAS, 479, 776

Vilchez-Gomez, R., Pello, R., \& Sanahuja, B. 1994, A\&A, 283, 37

Vulcani, B., Poggianti, B. M., Aragón-Salamanca, A., et al. 2011, MNRAS, 412, 246

Wang, P., Guo, Q., Kang, X., \& Libeskind, N. I. 2018, ApJ, 866, 138

Willman, B., Governato, F., Wadsley, J., \& Quinn, T. 2004, MNRAS, 355, 159

Zeldovich, I. B., Einasto, J., \& Shandarin, S. F. 1982, Nature, 300, 407

Zenteno, A., Mohr, J. J., Desai, S., et al. 2016, MNRAS, 462, 830

Zhang, Y., Yang, X., Wang, H., et al. 2015, ApJ, 798, 17

Zhang, Y., Yanny, B., Palmese, A., et al. 2019, ApJ, 874, 165

Zwicky, F. 1951, PASP, 63, 61 